Pure and Applied Mathematics Quarterly

Volume 6, Number 3

(Special Issue: In honor of

Joseph J. Kohn)

$781-813,2010$

\title{
Weighted Bergman Kernels for Logarithmic Weights
}

\author{
Miroslav Engliš
}

Dedicated to Professor J.J.Kohn on the occasion of his 75th birthday

\begin{abstract}
We describe the boundary singularity of weighted Bergman kernels on smoothly bounded strictly pseudoconvex domains for weights which behave, roughly speaking, likely formal power series in defining function with coefficients which are polynomials in the logarithm of defining function. The result extends also to weighted Sobolev spaces of holomorphic functions with respect to such weights. Connections with holomorphic invariants are outlined, and some examples presented.
\end{abstract}

Keywords: Bergman kernel, Toeplitz operator, logarithmic weight, pseudodifferential operator

\section{INTRODUCTION}

Let $\Omega$ be a bounded strictly pseudoconvex domain in $\mathbf{C}^{n}$ with smooth boundary, and $r$ a defining function for $\Omega$; thus $r$ is smooth on the closure $\bar{\Omega}$ of $\Omega, r<0$ on $\Omega$, and $r=0,\|\nabla r\|>0$ on $\partial \Omega$. It is then a celebrated result of Fefferman [10] and Boutet de Monvel and Sjöstrand [6] that the Bergman kernel of $\Omega$ has the form

$$
K(x, y)=\frac{a(x, y)}{\rho(x, y)^{n+1}}+b(x, y) \log \rho(x, y)
$$

Received September 27, 2008.

1991 Mathematics Subject ClassificationPrimary 32W25; Secondary 32A36, 32A25, 47B35

Research supported by GA AV ČR grant no. IAA100190802 and Ministry of Education research plan no. MSM4781305904. 
for some functions $a, b \in C^{\infty}(\bar{\Omega} \times \bar{\Omega})$. Here $\rho(x, y)$ is a function in $C^{\infty}(\bar{\Omega} \times \bar{\Omega})$ which satisfies $\rho(x, x)=-r(x)$ and is almost-sesquianalytic in the sense that $\partial \rho(x, y) / \partial \bar{x}$ and $\partial \rho(x, y) / \partial y$ vanish to infinite order on the diagonal $x=y$. (It is known that such $\rho(x, y)$ always exists and can be chosen in such a way that $\rho(x, y)=\overline{\rho(y, x)}$ and

$$
2 \operatorname{Re} \rho(x, y) \geq c|x-y|^{2}+\rho(x)+\rho(y) \quad \forall x, y \in \bar{\Omega}
$$

for some $c>0$, so that, in particular, $\log \rho(x, y)$ and $\rho(x, y)^{\alpha}$ are well-defined functions in $C^{\infty}(\overline{\Omega \times \Omega} \backslash \operatorname{diag} \partial \Omega)$ for any $\alpha \in \mathbf{C}$. We will assume that such $\rho(x, y)$ has been chosen, from now on.) One has also the explicit formula

$$
a(x, x)=\frac{n !}{\pi^{n}} J[\rho](x), \quad \forall x \in \partial \Omega,
$$

for the boundary values of the function $a$, where $J[\rho]$ is the Monge-Ampere determinant

$$
J[\rho]=(-1)^{n} \operatorname{det}\left[\begin{array}{cc}
\rho & \partial \rho \\
\bar{\partial} \rho & \partial \bar{\partial} \rho
\end{array}\right]
$$

(whose positivity on $\partial \Omega$ follows from the strict pseudoconvexity of $\Omega$ ), and we are also abusing the notation slightly (and will continue to in the sequel) by using the same letter $\rho$ also for the single-variable function $\rho(x):=\rho(x, x)=-r(x)$.

If $w$ is a continuous positive weight on $\Omega$, one can consider also the weighted Bergman spaces $L_{\text {hol }}^{2}(\Omega, w)$ of all holomorphic functions in $L^{2}(\Omega, w)$, and their reproducing kernels, namely, the weighted Bergman kernels $K_{w}(x, y)$. That is, the function $K_{w, y}:=K_{w}(\cdot, y)$ belongs to $L^{2}(\Omega, w)$ for each $y \in \Omega$, and has the reproducing property that

$$
f(x)=\int_{\Omega} f(y) K_{w}(x, y) w(y) d y
$$

for all $x \in \Omega$ and $f \in L_{\text {hol }}^{2}(\Omega, w)$.

From the proof in [6], it is immediate that the formula (1) extends also to the weighted kernels $K_{w}(x, y)$ for smooth positive weights on $\bar{\Omega}$, i.e. for weights of the form $w=e^{g}$ with $g \in C^{\infty}(\bar{\Omega})$. Using an idea going back to Forelli and Rudin [11] (see also Ligocka [20] and Boas-Fu-Straube [1]), relating a weighted Bergman kernel of a domain to the unweighted Bergman kernel of a certain Hartogs domain over it, one can also obtain a generalization of (1) to weights on $\Omega$ of the form

$$
w=\rho^{m} e^{g}, \quad m=1,2,3, \ldots, g \in C^{\infty}(\bar{\Omega})
$$


The corresponding kernels $K_{w}$ turn out to be still of the form (1), only with the exponent $n+1$ in the denominator replaced by $n+m+1$.

Recently, the current author [9] was able to generalize the formula (1) also to weights behaving like a fractional power of the defining function - more precisely, to weights of the form

$$
w=\rho^{\alpha} e^{g}, \quad \alpha>-1, g \in C^{\infty}(\bar{\Omega}) .
$$

(The restriction on $\alpha$ stems from the fact that for $\alpha \leq-1$ the space $L_{\text {hol }}^{2}(\Omega, w)$ reduces to constant zero, and thus $K_{w} \equiv 0$ trivially.) The result is

$$
K_{w}(x, y)= \begin{cases}\frac{a(x, y)}{\rho(x, y)^{n+\alpha+1}}+b(x, y) \log \rho(x, y) & \text { if } \alpha \in \mathbf{Z}, \\ \frac{a(x, y)}{\rho(x, y)^{n+\alpha+1}}+b(x, y) & \text { if } \alpha \notin \mathbf{Z},\end{cases}
$$

again with some $a, b \in C^{\infty}(\bar{\Omega} \times \bar{\Omega})$ (depending on $w$ ). There is also an analogue of $(2)$,

$$
a(x, x)=\frac{\Gamma(n+\alpha+1)}{\pi^{n}} \frac{J[\rho](x)}{e^{g(x)}}, \quad \forall x \in \partial \Omega
$$

Finally, it was also shown in [9] that an analogous result holds for the reproducing kernels of the holomorphic Sobolev spaces $W_{\text {hol }}^{s}(\Omega)$ of all holomorphic functions in the $s$-th order Sobolev space $W^{s}(\Omega)$, for any $s \in \mathbf{R}$. (Sometimes these are called Sobolev-Bergman spaces and kernels, cf. [16].) Namely,

(6) $K^{(s)}(x, y)= \begin{cases}\frac{a(x, y)}{\rho(x, y)^{n+1-2 s}}+b(x, y) & \text { if } n+1-2 s \notin \mathbf{Z}, \\ \frac{a(x, y)}{\rho(x, y)^{n+1-2 s}}+b(x, y) \log \rho(x, y) & \text { if } n+1-2 s \in \mathbf{Z}_{>0}, \\ \frac{a(x, y)}{\rho(x, y)^{n+1-2 s}} \log \rho(x, y)+b(x, y) & \text { if } n+1-2 s \in \mathbf{Z}_{\leq 0} .\end{cases}$

Again, there is also an analogue of (5), except that $\Gamma(n+\alpha+1)$ gets replaced by $\Gamma(n+1-2 s)$ if $n+1-2 s$ is not a nonpositive integer, and by $(-1)^{k+1} / k$ ! if $n+1-2 s=-k, k=0,1,2, \ldots$; and instead of $e^{g(x)}$ in the denominator there is a term whose exact form depends on the choice of the norm in $W^{s}(\Omega)$. We refer to [9] for the details.

While the class of weights covered by (4) is conveniently large, there are still many interesting cases that it misses. For instance, it does not contain weights 
like $u^{\alpha}, \alpha>-1$, where $u$ is the solution of the complex Monge-Ampere equation

$$
J[u]=1 \text { on } \Omega, \quad u=0,\|\nabla u\|>0 \text { on } \partial \Omega,
$$

because it is known (cf. Lee and Melrose [18]) that $u$ is not of the form (3), but rather

$$
u \approx \rho \sum_{j=0}^{\infty}\left(\rho^{n+1} \log \rho\right)^{j} \eta_{j}, \quad \eta_{j} \in C^{\infty}(\bar{\Omega}),
$$

with $\left.\eta_{0}\right|_{\partial \Omega}=J[\rho]^{-1 /(n+1)}$. Here " $\approx$ " means that the difference between the lefthand side and a partial sum of the right-hand side is continuous on $\bar{\Omega}$ together with as many derivatives and vanishes at $\partial \Omega$ to an order as high as the next term of the series, i.e.

$$
u-\rho \sum_{j=0}^{N-1}\left(\rho^{n+1} \log \rho\right)^{j} \eta_{j} \in C_{0}^{(n+1) N}(\bar{\Omega}), \quad \forall N=0,1,2, \ldots
$$

Our main result in this paper is the following generalization of Fefferman's formula (1), which covers also weights like (8).

Theorem 1. Let $\Omega$ be a smoothly bounded strictly pseudoconvex domain in $\mathbf{C}^{n}$ and $w>0$ a smooth weight on $\Omega$ of the form

$$
w \approx \rho^{\alpha} \sum_{k=0}^{\infty} \rho^{k} \sum_{j=0}^{N_{k}}(\log \rho)^{j} \eta_{j k}, \quad \eta_{j k} \in C^{\infty}(\bar{\Omega}), \alpha>-1, N_{k}<\infty,
$$

where $N_{0}=0$ and $\eta_{00}>0$ on $\partial \Omega$. Then

$$
K_{w} \approx \rho^{-n-\alpha-1} \sum_{k=0}^{\infty} \rho^{k} \sum_{j=0}^{M_{k}}(\log \rho)^{j} v_{j k}+v_{\infty}
$$

for some $M_{k}<\infty, M_{0}=0$, and $v_{j k}, v_{\infty} \in C^{\infty}(\bar{\Omega} \times \bar{\Omega})$. Furthermore,

$$
v_{00}(x, x)=\frac{\Gamma(n+\alpha+1)}{\pi^{n}} \frac{J[\rho](x)}{\eta_{00}(x)} \quad \forall x \in \partial \Omega .
$$

Here the asymptotic expansion in (9) is understood in the same sense as in (8), and similarly for (10), except that in the latter the continuity of (many) derivatives is meant on $\bar{\Omega} \times \bar{\Omega}$, while the vanishing (to high order) is meant only at diag $\partial \Omega$. If $\alpha$ is an integer, the term $v_{\infty}$ can be omitted. 
The numbers $M_{k}$ depend on the $N_{k}$ in a somewhat complicated manner which can be best described as follows. For $\epsilon>0$ small enough, the map

$$
\Psi: \partial \Omega \times(-\epsilon, \epsilon) \rightarrow \mathbf{C}^{n}, \quad(\zeta, t) \mapsto \zeta+t n_{\zeta},
$$

where $n_{\zeta}$ is the inward unit normal vector at $\zeta \in \partial \Omega$, is a diffeomorphism. Since $\partial r\left(\zeta+t n_{\zeta}\right) / \partial t=-\|\nabla r\|<0$ for $t=0$, it follows from the implicit function theorem that on a small neighbourhood of $\partial \Omega$ in $\mathbf{C}^{n}$ we can use $(\zeta, r)$ as local coordinates. Then (9) becomes

$$
w \approx \rho^{\alpha} \sum_{k=0}^{\infty} \rho^{k} \sum_{j=0}^{N_{k}}(\log \rho)^{j} \tau_{j k}
$$

for some $\tau_{j k} \in C^{\infty}(\partial \Omega)$. Consider now the ring $C^{\infty}(\partial \Omega)[\log \rho]$ of all polynomials in $\log \rho$ with coefficients in $C^{\infty}(\partial \Omega)$; and let $\mathcal{P}=C^{\infty}(\partial \Omega)[\log \rho][[\rho]]$ be the ring of all formal powers series in $\rho$ with coefficients in $C^{\infty}(\partial \Omega)[\log \rho]$. Then (12) says that, modulo functions in $C^{\infty}(\bar{\Omega})$ vanishing to infinite order at the boundary, $\rho^{-\alpha} w=: v$ can be identified with an element of $\mathcal{P}$; and the hypothesis that $N_{0}=0$ and $\left.\eta_{00}\right|_{\partial \Omega}>0$ means precisely that $v$ is a unit of $\mathcal{P}$, i.e. is invertible as a formal power series. Let $v^{-1} \in \mathcal{P}$ be the inverse of $v$,

$$
v^{-1}=\sum_{k=0}^{\infty} \rho^{k} \sum_{j=0}^{m_{k}}(\log \rho)^{j} \mu_{j k} .
$$

Then

$$
K_{w} \approx \sum_{k=0}^{\infty} \sum_{j=0}^{m_{k}} \rho^{[-n-\alpha-1+k]}(\log \rho)^{j} v_{j k}+v_{\infty}
$$

where

$$
\rho^{[\beta]}:= \begin{cases}\rho^{\beta}, & \text { if } \beta \in \mathbf{C} \backslash\{0,1,2, \ldots\}, \\ \rho^{\beta} \log \rho, & \text { if } \beta=0,1,2, \ldots\end{cases}
$$

Thus (10) holds with $M_{k}=\max _{0 \leq j \leq k} m_{j}+\epsilon_{k}$, where $\epsilon_{k}=0$ if $k-n-\alpha-1$ is not a nonnegative integer and $\epsilon_{k}=1$ otherwise.

In particular, we have the following corollary. For any integer $m \geq 1$, denote by $\mathcal{A}_{m}$ the subspace of all functions $v \in C^{\infty}(\Omega)$ of the form

$$
v \approx \sum_{j=0}^{\infty}\left(\rho^{m} \log \rho\right)^{j} \eta_{j}, \quad \eta_{j} \in C^{\infty}(\bar{\Omega}),
$$


and let $\mathcal{A}_{m}^{*}$ be the subset of all $v \in \mathcal{A}_{m}$ with $\left.\eta_{0}\right|_{\partial \Omega}>0$, i.e. $\left.v\right|_{\partial \Omega}>0$. Finally, abusing notation, we will denote by the same symbol $\mathcal{A}_{m}$ also the similar space on $\Omega \times \Omega($ with $\rho(x)$ replaced by $\rho(x, y)$ ).

Corollary 2. If

$$
w \in \rho^{\alpha} \mathcal{A}_{m}^{*}, \quad \alpha>-1, \alpha \notin \mathbf{Z},
$$

then

$$
K_{w} \in \rho^{-n-\alpha-1} \mathcal{A}_{m}^{*}+C^{\infty}(\bar{\Omega} \times \bar{\Omega}),
$$

with

$$
\rho^{n+\alpha+1} K_{w}=\frac{\Gamma(n+\alpha+1)}{\pi^{n}} \frac{J[\rho]}{\rho^{-\alpha} w}
$$

on the boundary diagonal.

Since $u \in \rho \mathcal{A}_{m}^{*}$ by (8), the last corollary applies e.g. to all weights of the form $u^{\alpha} e^{g}, \alpha>-1, g \in C^{\infty}(\bar{\Omega})$.

As in [9], the proof of Theorem 1 relies on Boutet de Monvel's and Guillemin's theory of Toeplitz operators on $\partial \Omega$ with pseudodifferential symbols. The main (and, essentially, the only) difficulty here is that for our present purpose we need to extend this theory from classical pseudodifferential operators to those with the more general "log-polyhomogeneous" symbols. This is done, along with a brief review of the necessary facts from Boutet de Monvel's and Guillemin's theory, in Section 2. After some preparatory work in Section 3, the proof of Theorem 1 appears in Section 4, together with an extension to weighted Sobolev-Bergman kernels. In the final Section 5, we present some examples, remarks on connections with holomorphic invariants, and possible further generalizations.

A short comment on notation and terminology: $\langle\cdot, \cdot\rangle_{\Omega},\langle\cdot, \cdot\rangle_{\Omega, w}$ and $\langle\cdot, \cdot\rangle_{\partial \Omega}$ stand for the inner products in $L^{2}(\Omega), L^{2}(\Omega, w)$ and $L^{2}(\partial \Omega)$, respectively; the subscripts are often omitted if there is no danger of confusion. Finally, by a positive operator on a Hilbert space we mean an operator $T$ such that $\langle T f, f\rangle>0$ for all $f \neq 0, f \in \operatorname{dom} T$. 


\section{Generalized Toeplitz operators}

Denote by $\eta$ the restriction to $\partial \Omega$ of the 1 -form $\operatorname{Im} \partial r=(\partial r-\bar{\partial} r) / 2 i$. The strict pseudoconvexity of $\Omega$ guarantees that $\eta$ is a contact form, i.e. the half-line bundle

$$
\Sigma:=\left\{(x, \xi) \in T^{*}(\partial \Omega): \xi=t \eta_{x}, t>0\right\}
$$

is a symplectic submanifold of $T^{*}(\partial \Omega)$. Equip $\partial \Omega$ with a measure with smooth positive density, and let $L^{2}(\partial \Omega)$ be the Lebesgue space with respect to this measure. The Hardy space $H^{2}(\partial \Omega)$ is the subspace in $L^{2}(\partial \Omega)$ of functions whose Poisson extension is holomorphic in $\Omega$; or, equivalently, the closure in $L^{2}(\partial \Omega)$ of $C_{\mathrm{hol}}^{\infty}(\partial \Omega)$, the space of boundary values of all the functions in $C^{\infty}(\bar{\Omega})$ that are holomorphic on $\Omega$. We will also denote by $W^{s}(\partial \Omega), s \in \mathbf{R}$, the Sobolev spaces on $\partial \Omega$, and by $W_{\text {hol }}^{s}(\partial \Omega)$ the corresponding subspaces of nontangential boundary values of functions holomorphic in $\Omega$. (Thus $W^{0}(\partial \Omega)=L^{2}(\partial \Omega)$ and $W_{\text {hol }}^{0}(\partial \Omega)=H^{2}(\partial \Omega)$.)

As usual, by a classical pseudodifferential operator or Fourier integral operator ( $\Psi$ DO or FIO for short) on $\partial \Omega$ we will mean an operator whose total symbol (or amplitude) in any local coordinate system has an asymptotic expansion

$$
p(x, \xi) \sim \sum_{j=0}^{\infty} p_{m-j}(x, \xi),
$$

where $p_{m-j}$ is $C^{\infty}$ in $x, \xi$, and is positive homogeneous of degree $m-j$ in $\xi$ for $|\xi|>1$. Here $j$ runs through nonnegative integers, but $m$ can be any complex number; and the symbol " $\sim$ " means that the difference between $p$ and $\sum_{j=0}^{k-1} p_{m-j}$ should belong to the Hörmander class $S^{\operatorname{Re} m-k}$, for each $k=0,1,2, \ldots$ The set of all classical $\Psi$ DOs on $\partial \Omega$ as above (i.e. of order $m$ ) will be denoted by $\Psi_{\mathrm{cl}}^{m}$. The (larger) class of all (not necessarily classical) $\Psi$ DOs whose total symbol in any local coordinate chart belongs to the Hörmander class $S^{m}=S_{1,0}^{m}, m \in \mathbf{R}$, will be denoted by $\Psi^{m}$; and we set, as usual, $\Psi_{\mathrm{cl}}:=\bigcup_{m \in \mathbf{C}} \Psi_{\mathrm{cl}}^{m}, \Psi:=\bigcup_{m \in \mathbf{R}} \Psi^{m}$, and $\Psi^{-\infty}:=\bigcap_{m \in \mathbf{C}} \Psi_{\mathrm{cl}}^{m}=\bigcap_{m \in \mathbf{R}} \Psi^{m}$. The operators in $\Psi^{-\infty}$ are precisely the smoothing operators, i.e. those given by a $C^{\infty}$ Schwartz kernel; and for any $P, Q \in \Psi$, we will write $P \sim Q$ if $P-Q$ is smoothing. Note that $\Psi_{\mathrm{cl}}^{m} \subset \Psi^{\operatorname{Re} m}$, and if $P \in \Psi^{m}$, then $P$ is continuous from $W^{s}(\partial \Omega)$ into $W^{s-m}(\partial \Omega)$, for any $s \in \mathbf{R}$. 
For $P \in \Psi^{m}$, the generalized Toeplitz operator $T_{P}: W_{\text {hol }}^{m}(\partial \Omega) \rightarrow H^{2}(\partial \Omega)$ is defined as

$$
T_{P}=\Pi P,
$$

where $\Pi: L^{2}(\partial \Omega) \rightarrow H^{2}(\partial \Omega)$ is the orthogonal projection (the Szegö projection). Alternatively, one may view $T_{P}$ as the operator

$$
T_{P}=\Pi P \Pi
$$

on all of $W^{m}(\partial \Omega)$. Actually, $T_{P}$ maps continuously $W^{s}(\partial \Omega)$ into $W_{\text {hol }}^{s-m}(\partial \Omega)$, for each $s \in \mathbf{R}$, because $\Pi$ is bounded on $W^{s}(\partial \Omega)$ for any $s \in \mathbf{R}$ (see [6]).

Microlocally, generalized Toeplitz operators have the following structure. Let $(x, y)$ denote the variable in $\mathbf{R}^{n} \times \mathbf{R}^{n-1} \simeq \mathbf{R}^{2 n-1}$, and let $(\xi, v)$ be the dual variable. We identify $T^{*} \mathbf{R}^{n}$ with the symplectic cone $\Sigma_{0} \subset T^{*} \mathbf{R}^{2 n-1}$ defined by $y=v=0$, and set

$$
D_{j}=\frac{\partial}{\partial y_{j}}+y_{j}\left|D_{x}\right|, \quad j=1, \ldots, n-1 .
$$

Let $H_{0}: C_{0}^{\infty}\left(\mathbf{R}^{n}\right) \rightarrow C^{\infty}\left(\mathbf{R}^{2 n-1}\right)$ be the Hermite operator

$$
H_{0} \phi(x, y)=(2 \pi)^{-n} \int_{\mathbf{R}^{n}} e^{i x \cdot \xi-\frac{1}{2}|\xi| y \cdot y}\left(\frac{|\xi|}{2 \pi}\right)^{(n-1) / 4} \hat{\phi}(\xi) d \xi
$$

where we write $x \cdot \xi$ for $\sum_{j} x_{j} \xi_{j}$, and the hat denotes Fourier transform; one has $D_{j} H_{0}=0$ for all $j$ and $H_{0}$ extends to an isometry from $L^{2}\left(\mathbf{R}^{n}\right)$ onto the subspace $\mathcal{H}_{0}:=\bigcap_{j=0}^{n-1} \operatorname{Ker} D_{j} \subset L^{2}\left(\mathbf{R}^{2 n-1}\right)$. Then it follows from [3] and [6] that $\Pi$ admits the following microlocal description: for any $z_{0} \in \partial \Omega$, there exists a homogeneous canonical transformation $\Phi$ from a conic open set $U \subset T^{*} \mathbf{R}^{2 n-1} \backslash\{0\}$ to a conic neighbourhood $V$ of $\left(z_{0}, \eta_{z_{0}}\right) \in \Sigma \subset T^{*} \partial \Omega \backslash\{0\}$, whose restriction defines a symplectic isomorphism $\chi: \Sigma_{0} \cap U \rightarrow \Sigma \cap V$. There exists an elliptic FIO $F$, defined in $U$ modulo smoothing operators, associated with $\Phi$, such that $F F^{*} \sim I$ on $V$ and $F^{*} \Pi F \sim\left(\right.$ the projection onto $\left.\mathcal{H}_{0}\right)$ on $U$, which transforms the left ideal of $\Psi$ DOs generated by the $D_{j}$ into the left ideal generated by the components of $\bar{\partial}_{b}$. Then $F H_{0}$ maps, modulo smoothing operators, $L^{2}\left(\mathbf{R}^{n}\right)$ (non-isometrically) onto $H^{2}(\partial \Omega)$. Set $A \sim H_{0}^{*} F^{*} F H_{0}$ (this is an elliptic positive classical $\Psi \mathrm{DO}$ ) and $H \sim F H_{0} A^{-1 / 2}$ (this is a classical FIO with complex phase, cf. [22]). Then $H^{*} H \sim I, H H^{*} \sim \Pi$, and for any $\Psi \mathrm{DO} Q$ on $\partial \Omega$,

$$
T_{Q}=\Pi Q \Pi \sim H P H^{*} \quad \text { near } z_{0} \text {, with } P \sim H^{*} Q H \sim H^{*} T_{Q} H .
$$

In fact the map $T_{Q} \mapsto P \sim H^{*} T_{Q} H$ is onto. It follows as a corollary that the generalized Toeplitz operators $T_{P}, P \in \Psi_{\mathrm{cl}}$, have the following properties. 
(P1) They form an algebra which is, modulo smoothing operators, locally isomorphic to the algebra of classical $\Psi$ DOs on $\mathbf{R}^{n}$.

(P2) In fact, for any $T_{P}$ there exists a $\Psi D O Q$ of the same order such that $T_{P}=T_{Q}$ and $Q \Pi=\Pi Q$.

(P3) It can happen that $T_{P}=T_{Q}$ for two different $\Psi$ DOs $P$ and $Q$. If $\operatorname{ord}(P)-$ $\operatorname{ord}(Q) \notin \mathbf{R}$, then $T_{P}=T_{R}$ for some $R \sim 0$. If $\operatorname{ord}(P)-\operatorname{ord}(Q)>0$, then the restriction of the principal symbol $\sigma(P)$ of $P$ to $\Sigma$ identically vanishes. If $\operatorname{ord}(P)=\operatorname{ord}(Q)$, then the restrictions of $\sigma(P)$ and $\sigma(Q)$ to the cone $\Sigma$ coincide. Thus we can define unambiguously the order of $T_{Q}$ as $\operatorname{ord}(Q)+\min \left\{\operatorname{ord}(P)-\operatorname{ord}(Q): T_{P}=T_{Q}\right\}$, and the symbol of $T_{Q}$ as $\sigma\left(T_{Q}\right):=\left.\sigma(Q)\right|_{\Sigma}$ if $\operatorname{ord}(Q)=\operatorname{ord}\left(T_{Q}\right)$.

(P4) The order and the symbol are multiplicative: $\operatorname{ord}\left(T_{Q} T_{Q^{\prime}}\right)=\operatorname{ord}\left(T_{Q}\right)+$ $\operatorname{ord}\left(T_{Q^{\prime}}\right)$ and $\sigma\left(T_{Q} T_{Q^{\prime}}\right)=\sigma\left(T_{Q}\right) \sigma\left(T_{Q^{\prime}}\right)$.

(P5) If $\operatorname{Re} \operatorname{ord}\left(T_{P}\right) \leq 0$, then $T_{P}$ is a bounded operator on $L^{2}(\partial \Omega)$; if $\operatorname{Re} \operatorname{ord}\left(T_{P}\right)<$ 0 , then it is even compact.

(P6) If $P \in \Psi_{\mathrm{cl}}^{m}$ and $\sigma\left(T_{P}\right)=0$, then there exists $Q \in \Psi_{\mathrm{cl}}^{m-1}$ with $T_{Q}=T_{P}$. In particular, if $T_{P} \sim 0$, then there exists a $\Psi$ DO $Q \sim 0$ such that $T_{P}=T_{Q}$.

(P7) We will say that a generalized Toeplitz operator $T_{P}$ of order $m$ is elliptic if $\sigma\left(T_{P}\right)$ does not vanish. Then $T_{P}$ has a parametrix, i.e. there exists a Toeplitz operator $T_{Q}$ of order $-m$, with $\sigma\left(T_{Q}\right)=\sigma\left(T_{P}\right)^{-1}$, such that $T_{P} T_{Q} \sim I_{H^{2}(\partial \Omega)} \sim T_{Q} T_{P}$.

We refer to the book [5], especially its Appendix, and to the paper [4] (which we have loosely followed in this section) for the proofs and additional information on generalized Toeplitz operators.

In addition to classical $\Psi$ DOs, we will need the more general class $\Psi_{\log }$ of log-polyhomogeneous $\Psi$ DOs, whose total symbol in any local coordinates satisfies

$$
p(x, \xi) \sim \sum_{j=0}^{\infty} p_{m-j}(x, \xi)
$$

where $p_{m-j}$ is of the form

$$
p_{m-j}(x, \xi)=\sum_{k=0}^{k_{j}} p_{m-j, k}\left(x, \frac{\xi}{|\xi|}\right)|\xi|^{m-j}(\log |\xi|)^{k}
$$


for $|\xi|>1$, for some (finite) integers $k_{j}$. We denote the class of $\Psi$ DOs of this form $\Psi_{\log }^{m}$, and we again also set $\Psi_{\log }=\bigcup_{m \in \mathbf{C}} \Psi_{\log }^{m}$. Note that $\Psi_{\log }^{m} \subset \Psi^{\operatorname{Re} m+\epsilon}$ for any $\epsilon>0$; accordingly, the " $\sim$ " in (17) now means that $p-\sum_{j=0}^{N-1} p_{m-j} \epsilon$ $\Psi^{\operatorname{Re} m-N+\epsilon}$ for any $\epsilon>0$ and $N=0,1,2, \ldots$ We will call $P$ pure if $k_{0}=0$, and pure elliptic if $k_{0}=0$ and $p_{m}(x, \xi) \neq 0$ for $\xi \neq 0$. More generally, we will denote by $\Psi_{\log }^{m, k}$ the class of all $\Psi$ DOs with symbol of the form (17) where $k_{0}=k$; so pure symbols correspond to $\Psi_{\log }^{m, 0}$. For $P \in \Psi_{\log }^{m}$ such that $p_{m}$ does not vanish identically, we still call $m=$ : $\operatorname{ord}(P)$ the order of $P$ (as before, this can be any complex number), and $p_{m}=: \sigma(P)$ the (principal) symbol of $P$; this clearly agrees with the corresponding notions for classical $\Psi$ DOs.

The standard reference for log-polyhomogeneous $\Psi$ DOs is Schrohe [26]; see also Lesch [19], Okikiolu [23], Grubb [13], and Paycha and Scott [25]. For the various closely related topics like complex powers and logarithms of $\Psi$ DOs, holomorphic families of $\Psi$ DOs, etc., see also Ouedraogo and Paycha [24], Grubb [13], Bucicovschi [7], and the book by Shubin [28].

The properties (P1)-(P7) above were established in the [5] and [4] only for classical $\Psi$ DOs. To extend them also to the present setting of log-polyhomogeneous symbols, one could in principle go through and check all the relevant technical details in those two references; but it is easier to use instead the fact that operators in $\Psi_{\log }$ are, essentially, the logarithms of complex powers of classical $\Psi$ DOs. More precisely, each operator in $\Psi_{\log }^{m, k}$ arises, modulo lower order terms, as $\left(\frac{\partial}{\partial z}\right)^{k} A^{z}$ for some $A \in \Psi_{\mathrm{cl}}^{m}$.

Recall that if $A$ is a positive selfadjoint elliptic classical $\Psi D O$ of order $m>0$ on $\partial \Omega$, then $A^{-1}$ is compact, hence its spectrum consists of isolated eigenvalues $0<\lambda_{1}<\lambda_{2}<\ldots$ of finite multiplicity. We can therefore define for any $z \in \mathbf{C}$ the operator $A^{z}$ by the spectral theorem, i.e.

$$
A^{z}=\sum_{j} \lambda_{j}^{z} P_{j}
$$

where $P_{j}$ is the projection onto the eigenspace corresponding to $\lambda_{j}$. Alternatively, one can define $A^{z}$ for $\operatorname{Re} z<0$ by the contour integral

$$
A^{z}=\oint_{\lambda_{1} / 2-i \infty}^{\lambda_{1} / 2+i \infty} \lambda^{z}(A-\lambda)^{-1} d \lambda
$$


(with the branch of $\lambda^{z}$ defined in the right half-plane so that $1^{z}=1$ ). For $\operatorname{Re} z \geq$ 0 , one then sets

$$
A^{z}=A^{k} A^{z-k}, \quad k>\operatorname{Re} z
$$

this is unambiguous since $A A^{z}=A^{z+1}$ for $\operatorname{Re} z<-1$. For a positive self-adjoint elliptic classical $\Psi$ DO of degree $m<0$, one then defines $A^{z}$ as $\left(A^{-1}\right)^{-z}$, the righthand side being defined as above. In both cases $(m<0$ and $m>0)$, the operator $A^{z}$ so defined is normal for any $z \in \mathbf{C}$, and self-adjoint and positive if $z$ is real.

It is then a result going back to Seeley [27] (see also Shubin [28], Bucicovschi [7] or Schrohe [26]), that the operator $A^{z}$ defined as above is again a classical $\Psi \mathrm{DO}$, of order $m z$, and with symbol $\sigma(A)^{z}$. Furthermore, the total symbol of $A^{z}$, in any local coordinate system, depends holomorphically on $z$ (i.e. each $(m z-j)$ th homogeneous component does).

Differentiating with respect to $z$, we also see that for any $k=0,1,2, \ldots$,

$$
A^{z}(\log A)^{k}=\sum_{j} \lambda_{j}^{z}\left(\log \lambda_{j}\right)^{k} P_{j}
$$

is an operator in $\Psi_{\log }^{m z, k}$, with principal symbol $\sigma(A)^{z}(\log \sigma(A))^{k}$.

Proposition 3. Generalized Toeplitz operators $T_{P}$ with log-polyhomogeneous $P \in$ $\Psi_{\log }$ have the seven properties listed right below.

(P1) They form an algebra which is, modulo smoothing operators, locally isomorphic to the algebra of log-polyhomogeneous $\Psi$ DOs on $\mathbf{R}^{n}$.

(P2) In fact, for any $T_{P}, P \in \Psi_{\log }^{m, k}$, there exists $Q \in \Psi_{\log }^{m, k}$ such that $T_{P}=T_{Q}$ and $Q \Pi=\Pi Q$.

(P3) It can happen that $T_{P}=T_{Q}$ for two different $\Psi$ DOs $P$ and $Q$. If $\operatorname{ord}(P)-$ $\operatorname{ord}(Q) \notin \mathbf{R}$, then $T_{P}=T_{R}$ for some $R \sim 0$. If $\operatorname{ord}(P)-\operatorname{ord}(Q)>0$, then the restriction of the principal symbol $\sigma(P)$ of $P$ to $\Sigma$ identically vanishes. If $\operatorname{ord}(P)=\operatorname{ord}(Q)$, then the restrictions of $\sigma(P)$ and $\sigma(Q)$ to the cone $\Sigma$ coincide. Thus we can define unambiguously the order of $T_{Q}$ as $\operatorname{ord}(Q)+\min \left\{\operatorname{ord}(P)-\operatorname{ord}(Q): T_{P}=T_{Q}\right\}$, and the symbol of $T_{Q}$ as $\sigma\left(T_{Q}\right):=\left.\sigma(Q)\right|_{\Sigma}$ if $\operatorname{ord}(Q)=\operatorname{ord}\left(T_{Q}\right)$.

(P4) The order and the symbol are multiplicative: $\operatorname{ord}\left(T_{Q} T_{Q^{\prime}}\right)=\operatorname{ord}\left(T_{Q}\right)+$ $\operatorname{ord}\left(T_{Q^{\prime}}\right)$ and $\sigma\left(T_{Q} T_{Q^{\prime}}\right)=\sigma\left(T_{Q}\right) \sigma\left(T_{Q^{\prime}}\right)$.

(P5) If $\operatorname{Re} \operatorname{ord}(P)=0$ and $P$ is pure, then $T_{P}$ is a bounded operator on $L^{2}(\partial \Omega)$; if $\operatorname{Re} \operatorname{ord}\left(T_{P}\right)<0$, then it is even compact. 
(P6) If $P \in \Psi_{\log }^{m}$ and $\sigma\left(T_{P}\right)=0$, then there exists $Q \in \Psi_{\log }^{m-1}$ with $T_{Q}=T_{P}$. In particular, if $T_{P} \sim T_{Q}$, then there exists a $\Psi D O R \sim 0$ such that $T_{P}-T_{Q}=T_{R}$.

(P7) We will say that a generalized Toeplitz operator $T_{P}$ is pure elliptic if $P$ is pure and $\sigma\left(T_{P}\right)$ does not vanish. (Note that, by (P3), this implies $\operatorname{ord}\left(T_{P}\right)=\operatorname{ord}(P) \neq-\infty$.) Then $T_{P}$ has a parametrix, i.e. there exists a pure elliptic Toeplitz operator $T_{Q}$ of order $-\operatorname{ord}\left(T_{P}\right)$, with $\sigma\left(T_{Q}\right)=$ $\sigma\left(T_{P}\right)^{-1}$, such that $T_{P} T_{Q} \sim I_{H^{2}(\partial \Omega)} \sim T_{Q} T_{P}$.

Proof. All these are similar and we will just look at (P2). Consider first $P \in \Psi_{\log }^{m}$ such that $p_{m}(x, \xi)=p_{m, l}\left(x, \frac{\xi}{|\xi|}\right)|\xi|^{m}(\log |\xi|)^{l}$ for $|\xi|>1$. Let $P_{1} \in \Psi_{\mathrm{cl}}^{m}$ be any classical $\Psi D O$ whose principal symbol is $p_{m, l}\left(x, \frac{\xi}{|\xi|}\right)|\xi|^{m}$ for $|\xi|>1$. By the property (P2) for classical operators, there exists $Q_{1} \in \Psi_{\mathrm{cl}}^{m}$ such that $T_{P_{1}}=T_{Q_{1}}$ and $\left[Q_{1}, \Pi\right]=0$; also, $\sigma\left(P_{1}\right)=\sigma\left(Q_{1}\right)$ on $\Sigma$. Similarly, there exists $\Upsilon \in \Psi_{\mathrm{cl}}^{1}$ such that $[\Upsilon, \Pi]=0$ and $\sigma(\Upsilon)=|\xi|$ on $\Sigma$. Arguing as in the proof of Proposition 16 in [9], we can in fact assume that $\Upsilon$ is in addition elliptic, selfadjoint and injective. Thus it has complex powers $\Upsilon^{z}, z \in \mathbf{C}$, and

$$
T_{P_{1} \Upsilon^{z}}=\Pi P_{1} \Upsilon^{z} \Pi=\Pi P_{1} \Pi \Upsilon^{z}=\Pi Q_{1} \Pi \Upsilon^{z}=\Pi Q_{1} \Upsilon^{z} \Pi=T_{Q_{1} \Upsilon^{z}} .
$$

Differentiating $l$ times with respect to $z$ and setting $z=0$, we get

$$
P_{P_{1}(\log \Upsilon)^{l}}=T_{Q_{1}(\log \Upsilon)^{l}},
$$

where $\left[Q_{1}(\log \Upsilon)^{l}, \Pi\right]=0$ and $\left.\sigma\left(Q_{1}(\log \Upsilon)^{l}\right)\right|_{\Sigma}=\left.\sigma\left(P_{1}(\log \Upsilon)^{l}\right)\right|_{\Sigma}$.

Doing this for each $l=0,1, \ldots, k_{m}$ and proceeding inductively over $m$, we thus construct an operator $R \in \Psi_{\log }^{m, k}$ such that $[R, \Pi]=0$ and $T_{Q}-T_{R} \sim 0$. By the second part of the property (P6) (which in fact means the same for classical and $\log$-polyhomogeneous operators, since $\left.\Psi_{\mathrm{cl}}^{-\infty}=\Psi_{\log }^{-\infty}\right)$ and by the property (P2) applied to the smoothing (hence, again, classical) operator $T_{Q-R}$, there exists $S \sim 0$ such that $[S, \Pi]=0$ and $T_{S}=T_{Q-R}$. Set $P:=R+S$.

\section{Reproducing Kernels on the Hardy SPACE}

The Hardy space $H^{2}(\partial \Omega)$ also has a reproducing kernel, namely the Szegö kernel $S(x, y) \equiv S_{y}(x)=\overline{S_{x}(y)}, x, y \in \Omega$, which satisfies $S_{y} \in H^{2}(\partial \Omega) \forall y \in \Omega$ and

$$
f(x)=\left\langle f, S_{x}\right\rangle_{\partial \Omega}=\int_{\partial \Omega} f(y) S(x, y), \quad \forall x \in \Omega, f \in H^{2}(\partial \Omega),
$$


where, abusing the notation slightly, we are denoting by $f(x)$ the value at $x \in \Omega$ of the Poisson extension of $f$ from $\partial \Omega$ into the interior of $\Omega$. It turns out that $S$ again extends to be smooth up to the boundary of $\overline{\Omega \times \Omega}$ except for the boundary diagonal $\operatorname{diag} \partial \Omega=\{(x, x): x \in \partial \Omega\}$; more precisely, one has the analogue

$$
S(x, y)=\frac{a(x, y)}{\rho(x, y)^{n}}+b(x, y) \log \rho(x, y)
$$

of Fefferman's formula (1) (again with $a, b \in C^{\infty}(\bar{\Omega} \times \bar{\Omega})$ ), except the exponent $n+1$ in (1) is replaced by $n$. (See [6].)

It is convenient to view the boundary values $\left.S\right|_{\partial \Omega \times \partial \Omega}$ of $S(x, y)$ on $\partial \Omega \times \partial \Omega$ also in the distributional sense, i.e. as the limit for $\epsilon \searrow 0$ of $\left.S(x, y)\right|_{r(x)=r(y)=-\epsilon}$ in $C^{\infty}(\partial \Omega \times \partial \Omega)^{\prime}$. In this sense, $\left.S\right|_{\partial \Omega \times \partial \Omega}$ is a (classical) Fourier integral distribution which is the distributional kernel of the Szegö projector $\Pi: L^{2}(\partial \Omega) \rightarrow H^{2}(\partial \Omega)$.

The following theorem is modelled on Theorem 17 in [9].

Theorem 4. Let $T$ be a positive selfadjoint operator on $H^{2}(\partial \Omega)$ such that $T \sim T_{P}$ where $\sigma\left(T_{P}\right)>0$ and $P \in \Psi_{\log }^{2 s, 0}$ is pure elliptic and commutes with $\Pi$. Let $W_{\text {hol }}^{T}$ be the completion of $C_{\text {hol }}^{\infty}(\partial \Omega)$ with respect to the norm

$$
\|u\|_{T}^{2}:=\langle T u, u\rangle_{\partial \Omega} .
$$

Then

(a) $W_{\text {hol }}^{T}=W_{\text {hol }}^{\text {s }}(\partial \Omega)$, with equivalent norms;

(b) the reproducing kernel $K_{T}(x, y) \equiv K_{T, y}(x)$ of $W_{\text {hol }}^{T}$ satisfies

$$
\left.\left.K_{T}\right|_{\partial \Omega \times \partial \Omega} \sim\left(P^{-1} \otimes I\right) S\right|_{\partial \Omega \times \partial \Omega},
$$

where $P^{-1} \otimes I$ means that $P^{-1}$ applies to the first variable.

Here and below, " $f \sim g$ " for two elements of $C^{\infty}(\partial \Omega \times \partial \Omega)^{\prime}$ means that $f-g$ belongs to $C^{\infty}(\partial \Omega \times \partial \Omega)$.

Note that the existence of the complex powers $P^{z}, z \in \mathbf{C}$ - hence, in particular, of the $P^{-1}$ above and the $P^{-1 / 2}$ in the proof below - follows from the generalization of Seeley's theorem to $\Psi_{\log }^{2 s, 0}$ due to Schrohe [26]. (In the case of $P^{-1}$, we can, alternatively, since we are interested in $K_{T}$ only modulo smooth functions, also take instead of the inverse $P^{-1}$ any parametrix of $P$ guaranteed by the property (P7).) 
Proof. For any $u \in C_{\text {hol }}^{\infty}(\partial \Omega)(\subset \operatorname{dom} T)$,

$$
\|u\|_{T}^{2}=\langle T u, u\rangle_{\partial \Omega}=\left\|T^{1 / 2} u\right\|_{H^{2}(\partial \Omega)}^{2} .
$$

From $T \sim T_{P}$ we have $T^{1 / 2} \sim T_{P^{1 / 2}}=\left.P^{1 / 2}\right|_{H^{2}(\partial \Omega)}$, where $P^{1 / 2} \in \Psi_{\log }^{s, 0}$ is pure elliptic, commutes with $\Pi$ and $\sigma\left(P^{1 / 2}\right)>0$. By $(\mathrm{P} 7), T_{P^{1 / 2}}$ has a parametrix, hence is Fredholm as an operator from $W_{\text {hol }}^{s}(\partial \Omega)$ into $H^{2}(\partial \Omega)$. The same is therefore true for $T^{1 / 2}$; since we know $T$, and, hence, $T^{1 / 2}$ to be positive (hence, injective) and selfadjoint as an operator on $H^{2}(\partial \Omega)$, it follows that $T^{1 / 2}$ is an isomorphism of $W_{\text {hol }}^{s}(\partial \Omega)$ onto $H^{2}(\partial \Omega)$. This proves (a).

For (b), note that for each $y \in \Omega$ and $f \in C_{\text {hol }}^{\infty}(\partial \Omega)$,

$$
\left\langle f, T^{-1} S_{y}\right\rangle_{T}=\left\langle T f, T^{-1} S_{y}\right\rangle=\left\langle f, S_{y}\right\rangle=f(y)=\left\langle f, K_{T, y}\right\rangle_{T} .
$$

Here the ultimate left-hand side makes sense since $T^{-1} S_{y} \in C_{\mathrm{hol}}^{\infty}(\partial \Omega) \subset W_{\text {hol }}^{T}$ by the preceding paragraph. It follows that $\left.K_{T, y}\right|_{\partial \Omega}=T^{-1}\left(\left.S_{y}\right|_{\partial \Omega}\right)$ for each $y \in \Omega$. Passing again to the boundary values in the distributional sense, this means that $\left.K_{T}\right|_{\partial \Omega \times \partial \Omega}$ is the distributional kernel of the operator $T^{-1} \Pi$. As $T^{-1} \Pi \sim P^{-1} \Pi$, part (b) follows.

Theorem 5. Let $T$ be a positive self-adjoint operator on $H^{2}(\partial \Omega)$ such that $T \sim$ $T_{Q}$ where $Q \in \Psi_{\log }^{2 s, 0}$ and $\sigma\left(T_{Q}\right)>0$, and let $W_{\text {hol }}^{T}$ and $K_{T}$ have the same meaning as in the preceding theorem. Then $K_{T}$ has the form

$$
K_{T} \approx \sum_{k=0}^{\infty} \rho^{[k+2 s-n]} \sum_{j=0}^{M_{k}}(\log \rho)^{j} v_{j k}+v_{\infty},
$$

where $M_{k}<\infty, M_{0}=0, v_{j k}, v_{\infty} \in C^{\infty}(\bar{\Omega} \times \bar{\Omega})$, and $\rho^{[\beta]}$ has the same meaning as in (15). Furthermore,

$$
v_{00}(x, x)= \begin{cases}\frac{\Gamma(n-2 s)}{\pi^{n}} \frac{J[\rho](x)}{\sigma\left(T_{Q}\right)(x, \partial r(x))} & \text { if } n-2 s \in \mathbf{C} \backslash\{0,-1,-2, \ldots\}, \\ \frac{(-1)^{k+1}}{k ! \pi^{n}} \frac{J[\rho](x)}{\sigma\left(T_{Q}\right)(x, \partial r(x))} & \text { if } n-2 s=-k, k=0,1,2, \ldots\end{cases}
$$

Proof. By (P2), we have $T_{Q}=T_{P}$ for some $P$ which has the same properties as $Q$ and moreover commutes with $\Pi$; to this $P$, the preceding theorem applies. Using 
the well-known formulas

$$
\int_{1}^{\infty} e^{-t p} t^{s} d t= \begin{cases}\frac{\Gamma(s+1)}{p^{s+1}}+\mathcal{O}(p), & s \in \mathbf{C} \backslash\{-1,-2, \ldots\}, \\ \frac{(-1)^{k+1}}{k !} p^{k}(\log p+\mathcal{O}(p)), & s=-1-k, k \in \mathbf{Z}_{\geq 0},\end{cases}
$$

valid for $\operatorname{Re} p>0$, where $\mathcal{O}(p)$ denotes a function of $p$ which is smooth (in fact - holomorphic) in a neighbourhood of the origin, the boundary singularity (18) of $S$ can also be rewritten as

$$
S(x, y) \sim \int_{0}^{\infty} e^{-t \rho(x, y)} b(x, y, t) d t, \quad x, y \in \bar{\Omega}
$$

where $b$ is a classical symbol in $S^{n-1}\left(\bar{\Omega} \times \bar{\Omega} \times \mathbf{R}_{+}\right)$with asymptotic expansion

$$
b(x, y, t) \sim \sum_{j=0}^{\infty} t^{n-1-j} b_{j}(x, y) \quad \text { for } t>1,
$$

with some functions $b_{j} \in C^{\infty}(\bar{\Omega} \times \bar{\Omega})$. In other words,

$$
S \approx \int_{0}^{\infty} \sum_{j=0}^{\infty} t^{n-1-j} e^{-t \rho} b_{j} d t
$$

where " $\approx$ " has the same meaning as in the Introduction. Thus on $\partial \Omega \times \partial \Omega$

$$
\left(P^{-1} \otimes I\right) S \sim \int_{0}^{\infty} \sum_{j=0}^{\infty} t^{n-1-j}\left(P^{-1} \otimes I\right)\left[e^{-t \rho} b_{j}\right] d t .
$$

Now by [26], $P^{-1}$ is an element of $\Psi_{\log }^{-2 s, 0}$, i.e. in any local coordinates has total symbol with asymptotic expansion

$$
\sum_{j=0}^{\infty} \sum_{m=0}^{M_{j}} a_{-2 s-j, m}\left(x, \frac{\xi}{|\xi|}\right)|\xi|^{-2 s-j}(\log |\xi|)^{m} \quad \text { for }|\xi|>1,
$$

with $M_{0}=0$ and $a_{-2 s, 0}\left(x, \frac{\xi}{|\xi|}\right)|\xi|^{-2 s}=\sigma\left(P^{-1}\right)(x, \xi)=\sigma(P)(x, \xi)^{-1}$. On the other hand, by the standard symbol calculus for $\Psi$ DOs (see, for instance, Theorem 4.2 in Hörmander [17]), we have quite generally for any classical $\Psi D O A$ of order $\alpha$

$$
t^{n-1-j}(A \otimes I)\left[e^{-t \rho} b_{j}\right] \sim t^{n-1-j+\alpha} e^{-t \rho} \sum_{l=0}^{\infty} b_{j, l} t^{-l}
$$

with some $b_{j, k} \in C^{\infty}(\partial \Omega \times \partial \Omega)$, where in particular

$$
b_{j, 0}(x, x)=t^{-\alpha} b_{j}(x, x) \sigma(P)\left(x,-\left.t \nabla_{x} \rho(x, y)\right|_{y=x}\right)=b_{j}(x, x) \sigma\left(T_{P}\right)(x, \partial r(x)) .
$$


By a similar argument as in the proof of Proposition 3 (i.e. invoking the complex powers $\Upsilon^{z}$ and taking logarithm), the formula (25) remains in force also for $A \in \Psi_{\log }^{\alpha, k}$ instead of $A \in \Psi_{\mathrm{cl}}^{\alpha}$, except that one gets also factors of $(\log t)^{m}, m=$ $0,1, \ldots, k$. Thus $(23)$ equals (modulo smooth functions on $\partial \Omega \times \partial \Omega$ )

$$
\int_{0}^{\infty} e^{-t \rho} \sum_{j=0}^{\infty} t^{n-1-j-2 s} \sum_{m=0}^{M_{j}}(\log t)^{m} \tilde{b}_{j, m} d t
$$

with the $M_{j}$ from (24) and some $\tilde{b}_{j, m} \in C^{\infty}(\partial \Omega \times \partial \Omega)$, where in particular

$$
\tilde{b}_{0,0}(x, x)=b_{0,0}(x, x)=b_{0}(x, x) \sigma\left(P^{-1}\right)(x, \partial r(x)) .
$$

Combining this with the generalization of the formulas (21) in the next lemma, and with the fact that $b_{0}(x, x)=J[\rho](x) / \pi^{n} \forall x \in \partial \Omega$ (cf. [6]), (19) and (20) follow.

Lemma 6. Let $m$ be a nonnegative integer. Then on $\operatorname{Re} p>0$,

$$
\int_{1}^{\infty} e^{-t p} t^{s}(\log t)^{m} d t=\mathcal{O}(p)+\sum_{j=0}^{m}\left(\begin{array}{c}
m \\
j
\end{array}\right) \frac{\Gamma^{(m-j)}(s+1)}{p^{s+1}}(-\log p)^{j}
$$

for $s \in \mathbf{C} \backslash\{-1,-2, \ldots\}$, and

$$
\int_{1}^{\infty} e^{-t p} t^{s}(\log t)^{m} d t=\mathcal{O}(p)+p^{k} \sum_{j=1}^{m+1} c_{j k m}(\log p)^{j}
$$

for $s=-k-1, k=0,1,2, \ldots$, with some constants $c_{j k m} \in \mathbf{R}$.

Proof. Let us denote the integrals on the left-hand side by $G_{s, m}(p)$, and set $G_{s}(p):=G_{s, 0}(p)$. Clearly

$$
G_{s, m}(p)=\left(\frac{d}{d s}\right)^{m} G_{s}(p)
$$

and

$$
\frac{d}{d p} G_{s, m}(p)=-G_{s+1, m}(p)
$$


For $\operatorname{Re} s>-1$, we have

$$
\begin{aligned}
G_{s}(p) & =\frac{1}{p^{s+1}} \int_{p}^{\infty} e^{-t} t^{s} d t \\
& =\frac{1}{p^{s+1}}\left[\Gamma(s+1)-\int_{0}^{p} e^{-t} t^{s} d t\right] \\
& =\frac{1}{p^{s+1}}\left[\Gamma(s+1)-\sum_{j=0}^{\infty} \frac{p^{j+s+1}(-1)^{j}}{j !(j+s+1)}\right] \\
& =\frac{\Gamma(s+1)}{p^{s+1}}-\sum_{j=0}^{\infty} \frac{(-p)^{j}}{j !(j+s+1)} .
\end{aligned}
$$

Applying (28) to both sides yields

$$
G_{s, m}(p)=\left(\frac{d}{d s}\right)^{m} \frac{\Gamma(s+1)}{p^{s+1}}-\sum_{j=0}^{\infty} \frac{(-p)^{j}(-1)^{m} m !}{j !(j+s+1)^{m+1}},
$$

for $\operatorname{Re} s>-1$ and $m=0,1,2, \ldots$ The left-hand side is an entire function of $s$, while the right-hand side is holomorphic for $s \in \mathbf{C} \backslash\{-1,-2, \ldots\}$. By analytic continuation, the last equality thus remains in force for all $s \neq-1,-2, \ldots$, and applying the Leibniz rule to the first summand proves (26).

On the other hand, for $k=0,1,2, \ldots,(29)$ gives

$$
\begin{aligned}
G_{-k-1, m}(p) & =(-1)^{k+1} \underbrace{\int \ldots \int}_{k+1} G_{0, m}(p) d p \ldots d p \\
& =\mathcal{O}(p)+(-1)^{k+1} \underbrace{\int \ldots \int}_{k+1} \sum_{j=0}^{m}\left(\begin{array}{c}
m \\
j
\end{array}\right) \frac{\Gamma^{(m-j)}(1)}{p}(-\log p)^{j} d p \ldots d p \\
& =\mathcal{O}(p)+(-1)^{k+1} \sum_{j=0}^{m}\left(\begin{array}{c}
m \\
j
\end{array}\right) \frac{\Gamma^{(m-j)}(1)(-1)^{j}}{j+1} \underbrace{\int \ldots \int}_{k}(\log p)^{j+1} d p \ldots d p .
\end{aligned}
$$

Appealing to the recursion formula

$$
\int p^{k}(\log p)^{j} d p=\frac{p^{k+1}}{k+1} \sum_{l=0}^{j} \frac{(-1)^{j} j ! /(k+1)^{j}}{(-1)^{l} l ! /(k+1)^{l}}(\log p)^{l},
$$

which is easily proved by integrating by parts, (27) follows.

Remark. We have not tried to compute the constants $c_{j k m}$, although it is in principle possible from (30). 


\section{Weighted Bergman and Sobolev-Bergman kernels}

Let $\mathbf{K}$ denote the Poisson extension operator, i.e. $\mathbf{K}$ solves the Dirichlet problem

$$
\Delta \mathbf{K} u=0 \quad \text { on } \Omega,\left.\quad \mathbf{K} u\right|_{\partial \Omega}=u .
$$

(Thus $\mathbf{K}$ acts from functions on $\partial \Omega$ into functions on $\Omega$. Here $\Delta$ is the ordinary Laplace operator.) By the standard elliptic regularity theory (see e.g. [21]), $\mathbf{K}$ acts continuously from $W^{s}(\partial \Omega)$ onto the subspace $W_{\text {harm }}^{s+1 / 2}(\Omega)$ of all harmonic functions in $W^{s+1 / 2}(\Omega)$. In particular, it is continuous from $L^{2}(\partial \Omega)$ into $L^{2}(\Omega)$, and thus has a continuous Hilbert space adjoint $\mathbf{K}^{*}: L^{2}(\Omega) \rightarrow L^{2}(\partial \Omega)$. The composition

$$
\mathbf{K}^{*} \mathbf{K}=: \Lambda
$$

is known to be an elliptic positive classical $\Psi \mathrm{DO}$ on $\partial \Omega$ of order -1 . We have

$$
\Lambda^{-1} \mathbf{K}^{*} \mathbf{K}=I_{L^{2}(\partial \Omega)},
$$

while

$$
\mathbf{K} \Lambda^{-1} \mathbf{K}^{*}=\mathbf{\Pi}_{\text {harm }}
$$

the orthogonal projection in $L^{2}(\Omega)$ onto the subspace $L_{\text {harm }}^{2}(\Omega)$ of all harmonic functions. (Indeed, from (32) it is immediate that the left-hand side acts as the identity on the range of $\mathbf{K}$, while it trivially vanishes on $\operatorname{Ker} \mathbf{K}^{*}=(\operatorname{Ran} \mathbf{K})^{\perp}$.) Comparing (32) with (31), we also see that the restriction of $\Lambda^{-1} \mathbf{K}^{*}$ to $L_{\text {harm }}^{2}(\Omega)$ is the operator $\gamma$ of "taking the boundary values" of a harmonic function. Again, by elliptic regularity, $\gamma$ extends to a continuous operator from $W_{\text {harm }}^{s}(\Omega)$ onto $W^{s-1 / 2}(\partial \Omega)$, for any $s \in \mathbf{R}$, which is the inverse of $\mathbf{K}$.

The operators

$$
\Lambda_{w}:=\mathbf{K}^{*} w \mathbf{K},
$$

with $w$ a smooth function on $\Omega$, are governed by a calculus developed by Boutet de Monvel [2]. It was shown there that for $w$ of the form

$$
w=\rho^{\alpha} e^{g}, \quad \alpha>-1, g \in C^{\infty}(\bar{\Omega}),
$$

$\Lambda_{w}$ is an elliptic classical $\Psi D O$ on $\partial \Omega$ of order $-\alpha-1$, with symbol

$$
\sigma\left(\Lambda_{w}\right)(x, \xi)=\frac{\Gamma(\alpha+1)}{2|\xi|^{\alpha+1}} e^{g(x)}\|\partial r(x)\|^{\alpha} .
$$


In fact, [2] covered only the case of integer $\alpha>-1$, but the case of noninteger $\alpha$ can be treated in the same manner with no new difficulties, cf. the computation on the bottom of p. 256 and the remarks on the top of p. 257 in [4]. It is also immediate from this last computation that everything remains true in fact for any complex $\alpha, \operatorname{Re} \alpha>-1$, and moreover the total symbol of $\Lambda_{w}$ in any local coordinate system depends holomorphically on $\alpha$. Differentiating with respect to $\alpha$ we thus see that for

$$
w=\rho^{\alpha}(\log \rho)^{m} e^{g}, \quad \alpha>-1, g \in C^{\infty}(\bar{\Omega}), m=0,1,2, \ldots,
$$

we have

$$
\Lambda_{w} \in \Psi_{\log }^{-1-\alpha, m}
$$

with symbol

$$
\sigma\left(\Lambda_{w}\right)(x, \xi)=\frac{\Gamma(\alpha+1)}{2|\xi|^{\alpha+1}} e^{g(x)}\|\partial r(x)\|^{\alpha}\left(\log \frac{\|\partial r(x)\|}{|\xi|}\right)^{m} .
$$

With all these prerequisites in hand, we are ready to prove our main result.

Proof of Theorem 1. Let now $w$ be as in (9). For any $u, v \in C_{\text {hol }}^{\infty}(\partial \Omega)$,

$$
\begin{aligned}
\langle\mathbf{K} u, \mathbf{K} v\rangle_{\Omega, w} & =\langle w \mathbf{K} u, \mathbf{K} v\rangle_{\Omega} \\
& =\left\langle\mathbf{K}^{*} w \mathbf{K} u, v\right\rangle_{\partial \Omega} \\
& =\left\langle\Lambda_{w} u, v\right\rangle_{\partial \Omega} \\
& =\left\langle\Pi \Lambda_{w} u, v\right\rangle_{\partial \Omega} \\
& =\left\langle T_{\Lambda_{w}} u, v\right\rangle_{\partial \Omega} .
\end{aligned}
$$

Combining this with the findings from the previous paragraph, we see that $u \mapsto$ $\mathbf{K} u$ sets up a Hilbert space isomorphism between $L_{\text {hol }}^{2}(\Omega, w)$ and the space $W_{\text {hol }}^{T}$ of Theorem 4, where $T=T_{\Lambda_{w}}, \Lambda_{w} \in \Psi_{\log }^{-\alpha-1,0}$ and

$$
\sigma\left(\Lambda_{w}\right)=\frac{\Gamma(\alpha+1)}{2|\xi|^{\alpha+1}}\|\partial r(x)\|^{\alpha} \eta_{00}(x)>0,
$$

i.e. $\Lambda_{w}$ (hence, a fortiori, also $T_{\Lambda_{w}}$ ) is pure elliptic. It remains to apply Theorem 5; this gives (14) and, hence, (10). Finally, (20) yields (11).

Remark. In more detail, the derivation of the exact form of (14) (with the numbers $m_{k}$ from (13)) goes as follows. If $w$ is of the form (12), i.e.

$$
w \approx \rho^{\alpha} \sum_{k=0}^{\infty} \rho^{k} \sum_{j=0}^{N_{k}}(\log \rho)^{j}
$$


(we momentarily omit the various factors of $\tau_{j k} \in C^{\infty}(\partial \Omega)$, for brevity), then the Boutet de Monvel calculus says that $\Lambda_{w}$ has total symbol of the form

$$
\sigma_{\text {total }}\left(\Lambda_{w}\right) \sim|\xi|^{-1-\alpha} \sum_{k=0}^{\infty}|\xi|^{-k} \sum_{j=0}^{N_{k}}(\log |\xi|)^{j}
$$

(again, we temporarily omit the various factors of $a_{-\alpha-1-k, m}\left(x, \frac{\xi}{|\xi|}\right)$ and indicate just the orders of homogeneity in $\xi$ ). This is of the same form as the expansion (38) for $\rho w$ if we replace $\rho$ by $|\xi|^{-1}$. Now the standard construction of the parametrix amounts essentially to inverting (39) as a formal power series in $|\xi|$; consequently, if, by (13),

$$
w^{-1} \approx \rho^{-\alpha} \sum_{k=0}^{\infty} \rho^{k} \sum_{j=0}^{m_{k}}(\log \rho)^{j},
$$

then $\Lambda_{w}^{-1}$ must have total symbol of the form obtained from this by multiplying by $\rho^{-1}$ and substituting $|\xi|^{-1}$ for $\rho$ :

$$
\sigma_{\text {total }}\left(\Lambda_{w}^{-1}\right) \sim|\xi|^{\alpha+1} \sum_{k=0}^{\infty}|\xi|^{-k} \sum_{j=0}^{m_{k}}(\log |\xi|)^{j} .
$$

The same thus holds for the operator $P^{-1}$ in the proof of Theorem 5; and in terms of the integral representation (22) for the Szegö kernel, we get by the proof of Theorem 5

$$
\left(P^{-1} \otimes I\right) S \sim \int_{0}^{\infty} e^{-t \rho} B(t) d t
$$

where $B$ is of the same form, as far as degrees of (log-)homogeneity in $t$ are concerned, as when the $b(t)=\sum_{l=0}^{\infty} t^{n-1-l} b_{l}$ from (22) is multiplied by the righthand side of (40) with $|\xi|$ substituted by $t$. By the formulas in Lemma 6, (14) follows.

The extension to weighted Sobolev spaces of holomorphic functions is now straightforward. We present just one result of this kind; others can be obtained along the lines of Theorem 9, Corollary 14, Corollary 19 and Corollary 21 in [9].

Theorem 7. Consider the space $W_{\text {hol }}^{\#}(\Omega)$ of all holomorphic functions on $\Omega$ having finite norm

$$
\|f\|_{\#}^{2}:=\sum_{|\nu|=m}\left(\begin{array}{c}
m \\
\nu
\end{array}\right)\left\|\partial^{\nu} f\right\|_{\Omega, w}^{2}+\sum_{|\nu|<m}\left|\partial^{\nu} f\left(x_{0}\right)\right|^{2},
$$


with $w$ as in (9), and $x_{0}$ some fixed point of $\Omega$; here the first sum extends over all multiindices $\nu=\left(\nu_{1}, \ldots, \nu_{n}\right) \in \mathbf{Z}_{\geq 0}^{n}$ with $|\nu|:=\nu_{1}+\cdots+\nu_{n}=m$, and similarly for the second sum. Then $W_{h o l}^{\#}(\Omega)=W_{h o l}^{m-1 / 2}(\Omega)$ with equivalent norms, and the reproducing kernel $K^{\#}(x, y)$ of $W_{\text {hol }}^{\#}(\Omega)$ has the form (14) with $\alpha$ replaced by $\alpha-2 m$, and with some nonnegative finite integers $m_{k}, m_{0}=0$.

Proof. For any $u, v \in C_{\text {hol }}^{\infty}(\partial \Omega)$, we have

$$
\begin{aligned}
\langle\mathbf{K} u, \mathbf{K} v\rangle_{\#} & =\sum_{|\nu|=m}\left(\begin{array}{c}
m \\
\nu
\end{array}\right)\left\langle\partial^{\nu} \mathbf{K} u, \partial^{\nu} \mathbf{K} v\right\rangle_{\Omega, w}+\sum_{|\nu|<m} \partial^{\nu} \mathbf{K} u\left(x_{0}\right) \overline{\partial^{\nu} \mathbf{K} v\left(x_{0}\right)} \\
& \equiv\left\langle\Theta^{\prime} u, v\right\rangle+\left\langle\Theta^{\prime \prime} u, v\right\rangle .
\end{aligned}
$$

There exist differential operators $Z_{k}, k=1, \ldots, n$, on $\partial \Omega$ such that

$$
\gamma \partial_{k} f=Z_{k} \gamma f, \quad \forall f \in C_{\mathrm{hol}}^{\infty}(\bar{\Omega})
$$

(or $\partial_{k} \mathbf{K} u=\mathbf{K} Z_{k} u \forall u \in C_{\text {hol }}^{\infty}(\partial \Omega)$ ). Explicitly, one has

$$
Z_{k}=\partial_{k}-\sum_{j=1}^{n} \frac{r_{j} r_{k}}{\|\partial r\|^{2}} \bar{\partial}_{j},
$$

where for brevity we have introduced the notation

$$
r_{j}:=\partial_{j} r
$$

Thus we can write

$$
\begin{aligned}
\left\langle\partial^{\nu} \mathbf{K} u, \partial^{\nu} \mathbf{K} v\right\rangle_{\Omega, w} & =\left\langle w \partial^{\nu} \mathbf{K} u, \partial^{\nu} \mathbf{K} v\right\rangle_{\Omega} \\
& =\left\langle w \mathbf{K} Z^{\nu} u, \mathbf{K} Z^{\nu} v\right\rangle_{\Omega} \\
& =\left\langle Z^{* \nu} \mathbf{K}^{*} w \mathbf{K} Z^{\nu} u, v\right\rangle_{\partial \Omega} \\
& =\left\langle\Pi Z^{* \nu} \Lambda_{w} Z^{\nu} u, v\right\rangle_{\partial \Omega} .
\end{aligned}
$$

Summing over $\nu$ we conclude that

$$
\Theta^{\prime}=T_{Q}
$$

where

$$
Q=\sum_{|\nu|=m}\left(\begin{array}{c}
m \\
\nu
\end{array}\right) Z^{* \nu} \Lambda_{w} Z^{\nu}
$$


belongs to $\Psi_{\log }^{-\alpha-1+2 m, 0}$, and its principal symbol satisfies

$$
\begin{aligned}
\left.\sigma(Q)\right|_{\Sigma} & =\left.\sum_{|\nu|=m}\left(\begin{array}{c}
m \\
\nu
\end{array}\right) \overline{\sigma(Z)^{\nu}} \sigma(Z)^{\nu} \sigma\left(\Lambda_{w}\right)\right|_{\Sigma} \\
& =\left.|\xi|^{2 m} \sigma\left(\Lambda_{w}\right)\right|_{\Sigma},
\end{aligned}
$$

since $\left.\sigma\left(Z_{k}\right)\right|_{\Sigma}=\frac{|\xi|}{\|\partial r(x)\|} r_{k}$ (see [9], the proof of Theorem 8).

On the other hand,

$$
\Theta^{\prime \prime}=\sum_{|\nu|<m}\left\langle\cdot, P_{x_{0}}^{\nu}\right\rangle P_{x_{0}}^{\nu}
$$

where $P_{x_{0}}^{\nu}(\zeta):=\left.\bar{\partial}^{\nu} P(\zeta, \cdot)\right|_{x_{0}}$ is the derivative at $x_{0}$ of the Poisson kernel $P(\zeta, x)$. Since $P$ is known to be $C^{\infty}$ on $\partial \Omega \times \Omega, \Theta^{\prime \prime}$ is a smoothing operator.

Thus

$$
T:=\Theta^{\prime}+\Theta^{\prime \prime}=T_{Q}+\Theta^{\prime \prime} \sim T_{Q}
$$

satisfies $\langle\mathbf{K} u, \mathbf{K} v\rangle_{\#}=\langle T u, v\rangle_{\partial \Omega}$, and since $\Theta^{\prime}$ and $\Theta^{\prime \prime}$ are both self-adjoint and nonnegative (being sums of operators of the form $A^{*} A, A=\partial^{\nu} \mathbf{K}: H^{2}(\partial \Omega) \rightarrow$ $L^{2}(\Omega, w)$, and $\langle\cdot, f\rangle f, f \in H^{2}(\partial \Omega)$, respectively), while Ker $\Theta^{\prime}=$ \{polynomials of degree $<m\}$ whereas $\operatorname{Ker} \Theta^{\prime \prime}=\left\{\right.$ functions vanishing at $x_{0}$ to order at least $m$, $T$ is positive. It follows that $T \sim T_{Q}$ satisfies the hypothesis of Theorem 5 , and an application of the latter concludes the proof.

Remark. For $w$ of the form (34), the last theorem (and similar ones that can be obtained along the same lines) gives a strengthening of Theorem 8 of [9] (which required that $w$ have no logarithmic terms, and also required that the integer $m$ in our Theorem 7 be $<\alpha+1$ ), with a simpler proof.

\section{Appendix}

Let $\Pi: L^{2}(\Omega) \rightarrow L_{\text {hol }}^{2}(\Omega)$ be the Bergman projection. For any $w \in L^{\infty}(\Omega)$,

$$
\mathbf{T}_{w}:=\left.\Pi w\right|_{L_{\mathrm{hol}}^{2}(\Omega)}
$$

is the Toeplitz operator on the Bergman space $L_{\text {hol }}^{2}(\Omega)$ with symbol $w$; it can alternatively be also viewed as the operator $\Pi w \boldsymbol{\Pi}$ on the whole $L^{2}(\Omega)$ (upon extending by zero on $L^{2}(\Omega) \ominus L_{\text {hol }}^{2}(\Omega)$ ). The operator $\mathbf{T}_{w}$ also makes sense, as a 
densely defined (possibly unbounded) closable operator, even for any $w \in L^{1}(\Omega)$, by the recipe

$$
\mathbf{T}_{w} f(x):=\int_{\Omega} w f \bar{K}_{x}, \quad \forall f \in C_{\mathrm{hol}}^{\infty}(\bar{\Omega}) ;
$$

see Section 2 of [9].

It was established by Guillemin [14] and Boutet de Monvel [4], and used extensively e.g. in [9], that for $w$ of the form (34), the operator

$$
\mathcal{T}_{w}:=\left.\gamma \mathbf{\Pi} w \mathbf{K}\right|_{H^{2}(\partial \Omega)}
$$

obtained upon "transferring" $\mathbf{T}_{w}=\left.\mathbf{\Pi} w\right|_{L_{\mathrm{hol}}^{2}(\Omega)}$ to $H^{2}(\partial \Omega)$ by means of the operators $\mathbf{K}$ and $\gamma$, is actually (modulo smoothing operators) a generalized Toeplitz operator $T_{Q}$ with $Q$ a classical $\Psi$ DO on $\partial \Omega$ of order $-\alpha$ whose symbol can be explicitly computed. We show that this remains true also for our more general weights of the form (9), provided one allows for $Q$ log-polyhomogeneous $\Psi$ DOs. We begin with an auxiliary result.

Proposition 8. $\gamma \Pi \mathrm{\Pi K}=T_{\Lambda}^{-1} \Pi \Lambda$.

Proof. Set $\boldsymbol{\Pi}_{\Lambda}:=\mathbf{K} T_{\Lambda}^{-1} \Pi \Lambda \gamma$, an operator on $L_{\text {harm }}^{2}(\Omega)$; we need to show that $\Pi_{\Lambda}=\left.\boldsymbol{\Pi}\right|_{L_{\text {harm }}^{2}}$. Since $\Lambda \in \Psi_{\mathrm{cl}}^{-1}$ is elliptic, we have seen in course of the proof of Theorem 4 that $T_{\Lambda}^{-1}$ maps $W_{\text {hol }}^{s}(\partial \Omega)$ onto $W_{\text {hol }}^{s-1}(\partial \Omega)$, for any $s \in \mathbf{R}$; since $\Pi$ maps each $W^{s}(\partial \Omega)$ onto $W_{\text {hol }}^{s}(\partial \Omega)$, it thus follows that the range of $\Pi_{\Lambda}$ is $W_{\text {hol }}^{0}(\Omega)=L_{\text {hol }}^{2}(\Omega)$. Since $T_{\Lambda}^{-1} \Pi \Lambda$ acts as the identity on the range of $\Pi$, it is further immediate that $\boldsymbol{\Pi}_{\Lambda}^{2}=\boldsymbol{\Pi}_{\Lambda}$; thus $\boldsymbol{\Pi}_{\Lambda}$ is a projection of $L_{\text {harm }}^{2}(\Omega)$ onto $L_{\text {hol }}^{2}(\Omega)$. Finally, $\mathbf{\Pi}_{\Lambda}=\mathbf{K} T_{\Lambda}^{-1} \Pi \mathbf{K}^{*}=\mathbf{K} \Pi T_{\Lambda}^{-1} \Pi \mathbf{K}^{*}$ is evidently self-adjoint. So, indeed, $\Pi_{\Lambda}=\Pi$.

Corollary 9. Let $w$ be of the form (9), possibly with nonzero $N_{0}=m$ and with arbitrary $\eta_{00} \in C^{\infty}(\bar{\Omega})$. Then $\mathcal{T}_{w} \sim T_{Q}$, where $Q \in \Psi_{\log }^{-\alpha, m}$ and

$$
\sigma\left(T_{Q}\right)=\frac{\Gamma(\alpha+1)}{|\xi|^{\alpha}}\|\partial r(x)\|^{\alpha} \eta_{00}(x)\left(\log \frac{\|\partial r(x)\|}{|\xi|}\right)^{m} .
$$

Proof. By (32) and (33),

$$
\begin{aligned}
\gamma \mathbf{\Pi} w \mathbf{K} & =\gamma \mathbf{\Pi} \boldsymbol{\Pi}_{\text {harm }} w \mathbf{K} \\
& =\gamma \mathbf{\Pi} \mathbf{K} \Lambda^{-1} \mathbf{K}^{*} w \mathbf{K} .
\end{aligned}
$$


Using the last proposition, we thus get

$$
\gamma \mathbf{\Pi} w \mathbf{K}=T_{\Lambda}^{-1} \Pi \mathbf{K}^{*} w \mathbf{K}=T_{\Lambda}^{-1} \Pi \Lambda_{w}
$$

and

$$
\left.\gamma \mathbf{\Pi} w \mathbf{K}\right|_{H^{2}(\partial \Omega)}=T_{\Lambda}^{-1} T_{\Lambda_{w}} .
$$

Using (36), in combination with (35) for $w=1$ (thus giving the symbol of $\Lambda$ ), and the property (P4) yields the claim.

\section{EXAMPLES}

Recall that a domain functional is a map $\Omega \mapsto f_{\Omega}$ assigning to each bounded strictly pseudoconvex domain $\Omega \subset \mathbf{C}^{n}$ with smooth boundary a function $f_{\Omega}$ on $\Omega$. Examples of domain functionals are

$$
\Omega \mapsto K_{\Omega}(x):=K(x, x),
$$

the restriction to the diagonal of the Bergman kernel of $\Omega$ (with respect to the Lebesgue measure); or

$$
\Omega \mapsto S_{\Omega}(x):=S(x, x),
$$

the restriction to the diagonal of the Szegö kernel of $\Omega$ (with respect to the $(2 n-1)$-dimensional Hausdorff measure on $\partial \Omega)$.

The domain functional is said to be invariant of weight $\alpha, \alpha \in \mathbf{R}$, if

$$
f_{\Omega}=\left|J_{\phi}\right|^{2 \alpha /(n+1)} f_{\phi \Omega} \circ \phi
$$

for any biholomorphic map $\phi: \Omega \rightarrow \phi \Omega$; here $J_{\phi}$ denotes the complex Jacobian of $\phi$. For instance, the Bergman kernel $K_{\Omega}$ above is invariant of weight $n+1$. This follows from the well-known transformation rule for the Bergman kernel

$$
K_{\Omega}(x, y)=J_{\phi}(x) \overline{J_{\phi}(y)} K_{\phi \Omega}(\phi(x), \phi(y)) .
$$

The Szegö kernel $S_{\Omega}$ as defined above is not invariant, but can be made so upon using instead of the Hausdorff measure an appropriately chosen "invariant" surface element $\tau$ on $\partial \Omega$; namely, $\tau$ is uniquely determined by

$$
\tau \wedge d \rho=J[\rho]^{1 /(n+1)} d V \quad \text { on } \partial \Omega,
$$


where $d V$ is the Lebesgue volume in $\mathbf{C}^{n}$. Then $S_{\Omega}$ is of weight $n$. The solution $u=u_{\Omega}$ of the Monge-Ampere equation (7) is an invariant domain functional of weight -1 . The Bergman invariant

$$
\beta_{\Omega}:=\frac{\operatorname{det}\left[\partial \bar{\partial} \log K_{\Omega}\right]}{K_{\Omega}}
$$

is an invariant domain functional of weight 0 . More generally, if $f_{\Omega}$ is a nonvanishing invariant domain functional of any weight $\alpha \in \mathbf{R}$, then

$$
\Omega \mapsto \operatorname{det}\left[\partial \bar{\partial} \log f_{\Omega}\right]
$$

is always an invariant of weight $n+1$. Hence, for instance, the "Szegö invariant"

$$
\beta_{\Omega, \mathrm{Sz}}:=\frac{\operatorname{det}\left[\partial \bar{\partial} \log S_{\Omega}\right]}{S_{\Omega}^{(n+1) / n}}
$$

(with $S_{\Omega}$ the "invariantly" defined Szegö kernel) is of weight 0 . Another invariant of weight 0 is the scalar curvature of the Bergman metric,

$$
R_{\Omega}:=\Delta_{\text {Berg }} \log \operatorname{det}\left[\partial \bar{\partial} \log K_{\Omega}\right],
$$

where $\Delta_{\text {Berg }}$ is the Laplace-Beltrami operator with respect to the Bergman metric.

One can also get new invariants from old ones by means of weighted Bergman kernels.

Proposition 10. Let $f_{\Omega}$ be a positive domain functional which is invariant of weight $\alpha, \alpha \in \mathbf{R}$. Then the weighted Bergman kernel $K_{f_{\Omega}}(x, x)$ of $L^{2}\left(\Omega, f_{\Omega}\right)$ restricted to the diagonal is an invariant domain functional of weight $n+1-\alpha$.

Proof. If $\phi: \Omega \rightarrow \phi \Omega$ is a biholomorphism, then

$$
\begin{aligned}
K_{f_{\Omega}}^{\Omega} & =K_{\left|J_{\phi}\right|^{2 \alpha /(n+1)} f_{\phi \Omega} \circ \phi}^{\Omega} \\
& =\left|J_{\phi}\right|^{-\frac{2 \alpha}{n+1}} K_{f_{\phi \Omega} \circ \phi}^{\Omega} \\
& =\left|J_{\phi}\right|^{2-\frac{2 \alpha}{n+1}} K_{f_{\phi \Omega}}^{\phi \Omega} \circ \phi .
\end{aligned}
$$

Here the second equality used the fact that

$$
K_{|g|^{2} w}(x, y)=g(x)^{-1} \overline{g(y)^{-1}} K_{w}(x, y)
$$

for any zero-free holomorphic function $g$, and the third follows from the generalization

$$
K_{w \circ \phi}^{\Omega}(x, y)=J_{\phi}(x) \overline{J_{\phi}(y)} K_{w}^{\phi \Omega}(\phi(x), \phi(y))
$$


of the transformation rule (43).

Thus, for instance,

$$
\Omega \mapsto K_{u_{\Omega}^{\alpha}}(x, x), \quad \alpha>-1,
$$

is an invariant domain functional of weight $n+1+\alpha$. Similarly, $K_{\beta_{\Omega}}(x, x)$ is of weight $n+1$, and so is $K_{R_{\Omega}}(x, x)$; and $K_{K_{\Omega}^{1-\alpha /(n+1)}}(x, x)$ is of weight $\alpha$, for any $\alpha>n$.

For further discussion of invariant domain functionals, we refer to Hirachi and Komatsu [16] and Hirachi [15].

The boundary behaviour of the kernels from the last proposition for the various invariants $f_{\Omega}$ mentioned above can be obtained from the results in the preceding section. Recall that we have introduced the notation $\mathcal{A}_{m}, m=1,2, \ldots$, for the collection of all smooth functions on $\Omega$ of the form

$$
w \approx \sum_{j=0}^{\infty}\left(\rho^{m} \log \rho\right)^{j} \eta_{j}, \quad \eta_{j} \in C^{\infty}(\bar{\Omega}),
$$

with $\mathcal{A}_{m}^{*}$ the subset of those $w \in \mathcal{A}_{m}$ for which $\left.w\right|_{\partial \Omega}>0$ (i.e. $\left.\eta_{0}\right|_{\partial \Omega}>0$ ); and abusing the notation slightly we also used the same symbol for the analogous collection of functions on $\Omega \times \Omega$ (with $\rho$ interpreted then as $\rho(x, y)$ rather than $\rho(x)$ ).

Example 11. As was already noted in the Introduction,

$$
u \in \rho \mathcal{A}_{n+1}^{*},
$$

with $\left.(u / \rho)\right|_{\partial \Omega}=J[\rho]^{-1 /(n+1)}$. Thus for $\alpha>-1, w=u^{\alpha}$ is of the form (9), with $v:=\rho^{-\alpha} w=(u / \rho)^{\alpha} \in \mathcal{A}_{n+1}^{*}$.

Since $\mathcal{A}_{m}$ is, modulo functions vanishing to infinite order at $\partial \Omega$, isomorphic to the ring of formal power series $C^{\infty}(\bar{\Omega})\left[\left[\rho^{m} \log \rho\right]\right]$, with $\mathcal{A}_{m}^{*}$ corresponding precisely to the subgroup of its invertible elements, it follows that $v \in \mathcal{A}_{n+1}^{*}$ implies that also $v^{-1} \in \mathcal{A}_{n+1}^{*}$. By (14), we thus get

$$
K_{u^{\alpha}} \approx \sum_{j, k=0}^{\infty} \rho^{[-n-\alpha-1+j+(n+1) k]}(\log \rho)^{k} v_{k}+v_{\infty},
$$

with some $v_{k}, v_{\infty} \in C^{\infty}(\bar{\Omega} \times \bar{\Omega})$, where

$$
v_{0}(x, x)=\frac{\Gamma(n+\alpha+1)}{\pi^{n}} J[\rho](x)^{1+\alpha /(n+1)} \quad \forall x \in \partial \Omega .
$$


For $\alpha$ noninteger, this means that

$$
K_{u^{\alpha}} \in \rho^{-n-\alpha-1} \mathcal{A}_{n+1}^{*}+C^{\infty}(\overline{\Omega \times \Omega}),
$$

in full accordance with (16). For $\alpha=0,1,2, \ldots$, we get

$$
\begin{aligned}
K_{u^{\alpha}} \approx \rho^{-n-\alpha-1}\left(\sum_{(n+1) k+j<n+\alpha+1} \rho^{(n+1) k+j}(\log \rho)^{k} \eta_{k j}\right. & \\
& \left.+\sum_{(n+1) k+j \geq n+\alpha+1} \rho^{(n+1) k+j}(\log \rho)^{k+1} \eta_{k j}\right),
\end{aligned}
$$

with $\eta_{k j} \in C^{\infty}(\bar{\Omega} \times \bar{\Omega})$. If $\alpha=(n+1) a+b$ with $a, b \in \mathbf{Z}, a \geq 0,0 \leq b \leq n$, then we can rewrite (upon a small manipulation) the last formula as

$$
K_{u^{\alpha}} \in \rho^{-n-\alpha-1}\left(\mathcal{A}_{n+1}^{*}+\sum_{k=0}^{a+1} \rho^{n+\alpha+1}(\log \rho)^{k+1} \tau_{k}+\rho^{(n+1)(a+2)}(\log \rho)^{a+3} \mathcal{A}_{n+1}\right),
$$

with some $\tau_{k} \in C^{\infty}(\bar{\Omega} \times \bar{\Omega})$; hence,

$$
K_{u^{\alpha}} \in \rho^{-\alpha-n-1}\left(\mathcal{A}_{n+1}^{*}+\left(\rho^{n+1} \log \rho\right)^{a+1} \log \rho \mathcal{A}_{n+1}\right) .
$$

Example 12. It was shown by the present author [8] that

$$
\beta_{\Omega} \in \mathcal{A}_{n+1}^{*}, \quad \text { with }\left.\beta_{\Omega}\right|_{\partial \Omega}=\frac{(n+1)^{n} \pi^{n}}{n !} .
$$

Thus for $K_{\beta_{\Omega}}$ we get the same result as in the preceding example with $\alpha=0$, i.e.

$$
K_{\beta_{\Omega}} \in \rho^{-n-1}\left(\mathcal{A}_{n+1}^{*}+\rho^{n+1}(\log \rho)^{2} \mathcal{A}_{n+1}\right),
$$

with

$$
\left.\left(\rho^{n+1} K_{\beta_{\Omega}}\right)\right|_{\partial \Omega}=\frac{n !^{2} J[\rho]}{(n+1)^{n} \pi^{2 n}} .
$$

Similar result holds also for $K_{R_{\Omega}}$, since

$$
R_{\Omega} \in \mathcal{A}_{n+1}^{*},\left.\quad R_{\Omega}\right|_{\partial \Omega}=n,
$$

cf. Theorem $1(\mathrm{~g})$ in [8].

Example 13. More generally, for any $a, b, c \in \mathbf{R}$ with $b-(n+1) c>-1$,

$$
w=\beta_{\Omega}^{a} u_{\Omega}^{b} K_{\Omega}^{c}
$$

is of the form $\rho^{\alpha} \mathcal{A}_{n+1}^{*}$, with $\alpha=b-(n+1) c$, and $K_{w}$ has the form (44) if $\alpha \notin \mathbf{Z}$ or $(45)$ if $\alpha=0,1,2, \ldots$ 
Example 14. The invariantly defined Szegö kernel $S_{\Omega}$ satisfies

$$
S_{\Omega} \in \rho^{-n} \mathcal{A}_{n}^{*},\left.\quad\left(\rho^{n} S_{\Omega}\right)\right|_{\partial \Omega}=\frac{(n-1) !}{\pi^{n}} J[\rho]^{n /(n+1)} .
$$

A similar analysis as in Example 11 shows that for any $\alpha>-1$,

$$
K_{S_{\Omega}^{-\alpha / n}} \in \rho^{-n-\alpha-1} \mathcal{A}_{n}^{*}+C^{\infty}(\overline{\Omega \times \Omega})
$$

for $\alpha$ noninteger, and

$$
K_{S_{\Omega}^{-\alpha / n}} \in \rho^{-n-\alpha-1}\left(\mathcal{A}_{n}^{*}+\left(\rho^{n} \log \rho\right)^{a+1} \log \rho \mathcal{A}_{n}\right)
$$

if $\alpha=n a+b$, with $a, b$ integers, $a \geq 0,0 \leq b<n$.

Example 15. The kernel in this example is not an invariant domain functional, but involves higher order derivatives in a manner which is very natural from the geometric viewpoint (cf. the remark after this example). Consider the Hilbert space $\mathcal{H}$ of all holomorphic functions on $\Omega$ for which

$$
\|f\|_{\mathcal{H}}^{2}:=\int_{\Omega}|f|^{2}+\Delta_{\mathrm{Berg}}|f|^{2}
$$

is finite. Here as before

$$
\Delta_{\text {Berg }}=\sum_{j, k=1}^{n} g^{\bar{k} j} \partial_{j} \bar{\partial}_{k}
$$

denotes the Laplace-Beltrami operator with respect to the Bergman metric, i.e. $g^{\bar{k} j}$ is the inverse matrix to $g_{j \bar{k}}$,

$$
\sum_{l=1}^{n} g^{\bar{k} l} g_{l \bar{j}}=\delta_{k j}
$$

where

$$
g_{j \bar{k}}(z):=\frac{\partial^{2}}{\partial z_{j} \partial \bar{z}_{k}} \log K_{\Omega}(z)
$$

The same computation as in (41) shows that

$$
\langle\mathbf{K} u, \mathbf{K} v\rangle_{\mathcal{H}}=\left\langle T_{Q} u, v\right\rangle_{\partial \Omega},
$$

for any $u, v \in C_{\text {hol }}^{\infty}(\partial \Omega)$, where

$$
Q=\Lambda+\sum_{j, k=1}^{n} Z_{k}^{*} \Lambda_{g^{\bar{k} j}} Z_{j} .
$$


We have seen that $Z_{j}, Z_{k}^{*}$ are differential operators of order 1 , with symbols on $\Sigma$ given by $\frac{|\xi|}{\|\partial r\|} r_{j}$ and $\frac{|\xi|}{\|\partial r\|} \bar{r}_{k}$, respectively. On the other hand, it was shown in Theorem $1(\mathrm{~d})$ in [8] that

$$
g^{\bar{k} j} \in \rho \mathcal{A}_{n+1}, \text { with }\left.\left(\rho^{-1} g^{\bar{k} j}\right)\right|_{\partial \Omega}=-\frac{R^{\bar{k} j}}{n+1},
$$

where $\rho R^{\bar{k} j}$ is the inverse matrix to $\rho_{j \bar{k}}:=\partial_{j} \bar{\partial}_{k} \log \rho$; further, it transpires from Lemma 6, Proposition 7 and Lemma 8 in [8], and from the formula after (38) on page 35 there, that

$$
\sum_{j, k=1}^{n} g^{\bar{k} j} \bar{r}_{k} r_{j} \in \rho^{2} \mathcal{A}_{n+1}, \text { with }\left.\left(\frac{\sum_{j, k=1}^{n} g^{\bar{k} j} \bar{r}_{k} r_{j}}{\rho^{2}}\right)\right|_{\partial \Omega}=\frac{1}{n+1} .
$$

The first of these facts implies that $\sum_{j, k} Z_{k}^{*} \Lambda_{g^{\bar{k} j}} Z_{j}$ is a $\Psi D O$ on $\partial \Omega$ of order $\operatorname{ord}\left(Z_{k}^{*}\right)+\operatorname{ord}\left(Z_{j}\right)-1-\left(\right.$ the order of vanishing of $g^{\bar{k} j}$ at $\left.\partial \Omega\right)=1+1-1-1=0$, whose symbol restricted to $\Sigma$ is

$$
\left.\frac{|\xi|^{2}}{\|\partial r\|^{2}} \sum_{j, k=1}^{n} \bar{r}_{k} r_{j} \frac{g^{\bar{k} j}}{\rho}\right|_{\partial \Omega}=0
$$

- that is, the corresponding Toeplitz operator is in fact of order -1 ; and the second then implies that the principal symbol of this Toeplitz operator is simply $\frac{1}{n+1}|\xi|^{-1}$. Thus

$$
\operatorname{ord}\left(T_{Q}\right)=-1, \quad \sigma\left(T_{Q}\right)=1+\frac{1}{n+1},
$$

and the total symbol of $Q$ is of the same form as for $K_{w}$ in the proof of Theorem 1 with $w \in C^{\infty}(\bar{\Omega})+\rho^{n} \log \rho \mathcal{A}_{n+1} \subset \mathcal{A}_{n}^{*}$, where $\left.w\right|_{\partial \Omega}=\frac{n+2}{n+1}$. Applying Theorem 5 , we thus conclude that $\mathcal{H}=L_{\text {hol }}^{2}(\Omega)$ is in fact the Bergman space, and using (14) we get for the corresponding reproducing kernel

$$
K_{\mathcal{H}} \in \rho^{-n-1} \mathcal{A}_{n}^{*} .
$$

Remark. Note that the norm in $\mathcal{H}$ can be written as

$$
\|f\|_{\mathcal{H}}^{2}=\int_{\Omega}|f|^{2}+\left\|\nabla_{\operatorname{Berg}} f\right\|^{2}
$$

since for $f$ holomorphic, $\Delta_{\text {Berg }}|f|^{2}=\sum_{j, k} g^{\bar{k} j} \partial_{j} f \overline{\partial_{k} f}$ is the norm of the covariant derivative $\nabla_{\text {Berg }} f$ with respect to the Bergman metric. Thus, naively, one might view $\mathcal{H}$ as a "covariantly defined" first order holomorphic Sobolev space. That it turns out to coincide with the Bergman space, as the last example shows, is therefore perhaps mildly surprising. 
We remark that the same conclusion holds also for the variant when $\Delta_{\text {Poinc }}$, the Laplace-Beltrami operator with respect to the Poincare metric $g_{j \bar{k}}=\partial_{j} \bar{\partial}_{k} \log \frac{1}{u}$, is used instead of $\Delta_{\text {Berg. }}$.

At least for $\Omega$ the unit ball of $\mathbf{C}^{n}$, it seems that the same conclusion as in the last example is also obtained if one uses higher powers $\Delta^{m}|f|^{2}$ of the Laplacian, $m=2,3, \ldots$.

We conclude by mentioning one more extension of our method, namely, to weights of the form

$$
w \approx \sum_{j=0}^{\infty} \rho^{\alpha_{j}} \sum_{k=0}^{m_{j}}(\log \rho)^{k} \eta_{j k}, \quad \eta_{j k} \in C^{\infty}(\bar{\Omega}),
$$

with $\left.\eta_{00}\right|_{\partial \Omega}>0, m_{j}$ finite integers, $m_{0}=0$, and $\alpha_{j} \in \mathbf{R}$ satisfying $\alpha_{j} \nearrow+\infty$. In the language of formal series used in the Introduction, this admits the following description. For any ring $\mathcal{R}$ with identity $e$, let $\mathcal{R}\{\{\rho\}\}$ stand for the ring of all "power series with real exponents" over $\mathcal{R}$, i.e. of all formal sums

$$
R=\sum_{j=0}^{\infty} R_{j} \rho^{\alpha_{j}}, \quad R_{j} \in \mathcal{R}, \alpha_{j} \in \mathbf{R},
$$

where $\alpha_{j} \nearrow+\infty$. The last condition ensures that there are only finitely many $\alpha_{j}$ less than any given real number, and thus one can define addition and multiplication of such formal sums in the usual way. Finally, $e \rho^{0} \in \mathcal{R}\{\{\rho\}\}$ is the identity for $\mathcal{R}\{\{\rho\}\}$, and $R$ is invertible in $\mathcal{R}\{\{\rho\}\}$ if and only if $R_{0}$ is invertible in $\mathcal{R}$. With these notions, (46) can be rephrased as

$$
w \text { is an invertible element of } C^{\infty}(\partial \Omega)[\log \rho]\{\{\rho\}\}
$$

modulo functions in $C^{\infty}(\bar{\Omega})$ that vanish to infinite order at the boundary.

A computation similar to (37) then reveals that for any $u, v \in C_{\text {hol }}^{\infty}(\partial \Omega)$,

$$
\langle\mathbf{K} u, \mathbf{K} v\rangle_{\Omega, w}=\left\langle T_{Q} u, v\right\rangle_{\partial \Omega},
$$

where $Q$ is a $\Psi D O$ with total symbol of the form

$$
\sum_{j=0}^{\infty} \sum_{k=0}^{n_{j}} a_{-\beta_{j}, k}\left(x, \frac{\xi}{|\xi|}\right)|\xi|^{-\beta_{j}}(\log |\xi|)^{k} \quad \text { for }|\xi|>1,
$$

where $n_{j}<\infty, n_{0}=0, a_{-\beta_{0}, 0}>0$, and $\beta_{j}$ is the sequence obtained by rearranging the set $\left\{\alpha_{j}+m+1: j, m \geq 0\right\}$ in increasing order. It is easily checked that all the 
proofs in the preceding sections remain valid even for $\Psi$ DOs of this "multistep" form (see e.g. [26] for the complex powers of such operators), and, consequently, yield an analogue of (14) to the effect that if

$$
w^{-1} \approx \sum_{j=0}^{\infty} \rho^{\gamma_{j}} \sum_{k=0}^{k_{j}}(\log \rho)^{k} \mu_{k j}, \quad \mu_{k j} \in C^{\infty}(\partial \Omega),
$$

then

$$
K_{w} \approx \sum_{j=0}^{\infty} \rho^{\left[-n-1+\delta_{j}\right]} \sum_{k=0}^{n_{j}}(\log \rho)^{k} v_{k j}+v_{\infty},
$$

where $\delta_{j}$ is the sequence obtained upon rearranging the set $\left\{\gamma_{j}+l: j, l \geq 0\right\}$ in increasing order, $n_{j}=\max \left\{k_{i}: \gamma_{i}+l=\delta_{j}\right\}$, and $v_{k j}, v_{\infty} \in C^{\infty}(\bar{\Omega} \times \bar{\Omega})$. (These extra shifts by positive integers appear due to the fact that the symbol $b(x, y, t)$ for the Szegö kernel in (22) contains powers of $t$ increasing by one, and also the construction of parametrix of an elliptic $\Psi D O$ involves induction over orders going down with step one.) An extension to Sobolev-Bergman kernels is likewise straightforward.

Example 16. Let $w \in C^{\infty}(\Omega)$ be a positive weight function of the form

$$
w=\rho^{\alpha} w_{1}+w_{2}, \quad w_{1}, w_{2} \in C^{\infty}(\bar{\Omega}),
$$

where $0<\alpha<1$ and $\left.w_{2}\right|_{\partial \Omega}>0$. This is of the form (46) with $\alpha_{2 j}=j$, $\alpha_{2 j+1}=j+\alpha$. The inverse $w^{-1}$ is of the form (47) where $\gamma_{j}$ is obtained upon rearranging the set $\{l+k \alpha: l, k \geq 0\}$ in the increasing order. Consequently,

$$
K_{w} \approx \sum_{l, k=0}^{\infty} \rho^{[l+k \alpha-n-1]} v_{k l}, \quad v_{k l} \in C^{\infty}(\overline{\Omega \times \Omega}),
$$

or

$$
K_{w}=\sum_{0 \leq k<\frac{n+1}{\alpha}} \rho^{k \alpha-n-1} v_{k}+\sum_{\frac{n+1}{\alpha} \leq k<\infty} \rho^{k \alpha-n-1} v_{k} \log \rho
$$

for some $v_{k} \in C^{\infty}(\overline{\Omega \times \Omega})$, with $\left.v_{0}\right|_{\operatorname{diag} \partial \Omega}>0$.

\section{REFERENCES}

[1] H.P. Boas, S. Fu, E. Straube: The Bergman kernel function: explicit formulas and zeroes, Proc. Amer. Math. Soc. 127 (1999), 805-811

[2] L. Boutet de Monvel: Boundary problems for pseudo-differential operators, Acta Math. 126 (1971), 11-51 
[3] L. Boutet de Monvel: Hypoelliptic operators with double characteristics and related pseudodifferential operators, Comm. Pure Appl. Math. 27 (1974), 585-639

[4] L. Boutet de Monvel: On the index of Toeplitz operators in several complex variables, Invent. Math. 50 (1979), 249-272

[5] L. Boutet de Monvel, V. Guillemin: The spectral theory of Toeplitz operators, Ann. Math. Studies 99, Princeton University Press, Princeton, 1981

[6] L. Boutet de Monvel, J. Sjöstrand: Sur la singularité des noyaux de Bergman et de Szegö, Astérisque 34-35 (1976), 123-164

[7] B. Bucicovschi: An extension of the work of $V$. Guillemin on complex powers and zeta functions or elliptic pseudodifferential operators, Proc. Amer. Math. Soc. 127 (1999), 30813090

[8] M. Engliš: Boundary behaviour of the Bergman invariant and related quantities, Monatsh. Math. 154 (2008), 19-37

[9] M. Engliš: Toeplitz operators and weighted Bergman kernels, J. Funct. Anal. 255 (2008), 1419-1457

[10] C. Fefferman: The Bergman kernel and biholomorphic mappings of pseudoconvex domains, Inv. Math. 26 (1974), 1-65

[11] F. Forelli, W. Rudin: Projections on spaces of holomorphic functions in balls, Indiana Univ. Math. J. 24 (1974), 593-602

[12] G. Grubb: Trace defect formulas and zeta values for boundary problems, Traces in number theory, geometry and quantum fields (Bonn, 2005), pp. 137-153, Aspects Math. E38, Friedr. Viewegh, Weisbaden, 2008

[13] G. Grubb: On the logarithm component in trace defect formulas, Comm. Partial Diff. Equations 30 (2005), 1671-1716

[14] V. Guillemin: Toeplitz operators in n-dimensions, Integ. Eqs. Oper. Theory 7 (1984), 145205

[15] K. Hirachi: Invariant theory of the Bergman kernel of strictly pseudoconvex domains, Sugaku Expositions 17 (2004), 151-169

[16] K. Hirachi, G. Komatsu: Local Sobolev-Bergman kernels of strictly pseudoconvex domains, Analysis and geometry in several complex variables (Katata, 1997), pp. 63-96, Trends Math., Birkhauser, Boston, 1999

[17] L. Hörmander: Pseudo-differential operators, Comm. Pure Appl. Math. 18 (1965), 501-517

[18] J. Lee, R. Melrose: Boundary behaviour of the complex Monge-Ampére equation, Acta Math. 148 (1982), 159-192

[19] M. Lesch: On the noncommutative residue for pseudodifferential operators with logpolyhomogeneous symbols, Ann. Global Anal. Geom. 17 (1999), 151-187

[20] E. Ligocka: On the Forelli-Rudin construction and weighted Bergman projections, Studia Math. 94 (1989), 257-272

[21] J.-L. Lions, E. Magenes: Problèmes aux limites non homogènes et applications, vol. 1, Dunod, Paris, 1968 
[22] A. Melin, J. Sjöstrand: Fourier integral operators with complex valued phase functions, Fourier integral operators and partial differential equations, LNM 459, pp. 120-223, Springer Verlag, Berlin-Heidelberg, 1975

[23] K. Okikiolu: The multiplicative anomaly for determinants of elliptic operators, Duke Math. J. 79 (1995), 723-750

[24] M.-F. Ouedraogo, S. Paycha: The Campbell-Hausdorff formula for pseudodifferential operators revisited. Applications to determinants, preprint arXiv:math/0701076 (2007)

[25] S. Paycha, S. Scott: A Laurent expansion for regularized integrals of holomorphic symbols, Geom. Funct. Anal. 17 (2007), 491-536

[26] E. Schrohe: Complex powers of elliptic pseudodifferential operators, Integ. Eqs. Oper. Theory 9 (1986), 337-354

[27] R.T. Seeley: Complex powers of an elliptic operator, Singular Integrals, Proc. Symp. Pure Math. X, AMS, Providence, 1967, pp. 288-307

[28] M.A. Shubin, Pseudodifferential operators and spectral theory, Springer-Verlag, Berlin, 2001

Miroslav Engliš

Mathematics Institute

Silesian University at Opava, Na Rybníčku 1, 74601 Opava,

Czech Republic and Mathematics Institute,

Žitná 25, 11567 Prague 1, Czech Republic

E-mail: englis@math.cas.cz 